\title{
L. J. M. Bizeul et son « Aperçu général sur l'étude des voies romaines » : un jalon pour l'histoire de l'archéologie en Bretagne
}

Jacques Daniel

\section{(2) OpenEdition \\ Journals}

\section{Édition électronique}

URL : http://journals.openedition.org/abpo/3877

DOI : $10.4000 / a b p o .3877$

ISSN : 2108-6443

\section{Éditeur}

Presses universitaires de Rennes

\section{Édition imprimée}

Date de publication : 12 juillet 2018

Pagination : 193-231

ISBN : 978-2-7535-7604-9

ISSN : 0399-0826

Référence électronique

Jacques Daniel, « L. J. M. Bizeul et son « Aperçu général sur l'étude des voies romaines » : un jalon pour l'histoire de l'archéologie en Bretagne ", Annales de Bretagne et des Pays de l'Ouest [En ligne], 125-2 | 2018, mis en ligne le 12 juillet 2020, consulté le 06 janvier 2021. URL : http:// journals.openedition.org/abpo/3877 ; DOI : https://doi.org/10.4000/abpo.3877 


\title{
L. J. M. Bizeul et son "Aperçu général sur l'étude des voies romaines ": un jalon pour l'histoire de l'archéologie en Bretagne
}

\author{
Jacques DANIEL \\ Professeur agrégé, retraité de l'Éducation nationale
}

Au cours du deuxième congrès annuel de l'Association bretonne qui s'ouvrit à Rennes au mois de septembre 1844, Louis Jacques Marie Bizeul lut un Aperçu général sur l'étude des voies romaines ${ }^{1}$ qui retint l'attention des participants. Dans cette courte allocution que le congrès décida de faire imprimer, il retraça sa carrière. Évoquant ses premiers pas dans l'étude des antiquités, il souligna l'impulsion décisive qu'eut, pour ses recherches futures, sa rencontre, en 1820, avec Édouard Richer, l'auteur du très remarqué Voyage pittoresque dans le département de la LoireInférieure ${ }^{2}$. Elle le conduisit à concentrer ses recherches sur la période de l'occupation romaine des Gaules, qui, sur plus de quatre siècles, avait, pensait-il, profondément modifié la société antérieure. Mais, dit-il, les historiens tant anciens que modernes, ne lui ayant été d'aucun secours sur cette période, il en conclut " qu'il fallait laisser là les livres " et chercher les traces de cette histoire " armorico-romaine " sur le sol même de la Bretagne. Cheminant le long des voies antiques, il entreprit alors, " la carte de Cassini et le crayon à la main ", de dessiner la "géographie ancienne de la province ", autrement dit de restituer la trame viaire et le réseau urbain de l'Armorique romaine.

Un discours singulier, qui, en dépit de son caractère concis, se prête à plusieurs niveaux de lecture : replacé dans le contexte de la vie personnelle de L. J. M. Bizeul et à l'aune des enjeux du congrès de Rennes de

1. Bizeul, Louis Jacques Marie, "Aperçu général sur l'étude des voies romaines ", Bulletin de l'association bretonne, $\mathrm{n}^{\circ}$ 1, $2^{\mathrm{e}}$ partie, 1849, p. 3-9.

2. RICHER, Édouard, Voyages pittoresques dans le département de la Loire-Inférieure, Nantes, 1823. 
l'Association bretonne, ce texte permet d'éclairer singulièrement un moment particulier de l'histoire de l'archéologie en Bretagne ${ }^{3}$.

Figure 1 - L. J. M. Bizeul vers 1860 (Musée de Blain, archives Bizeul)

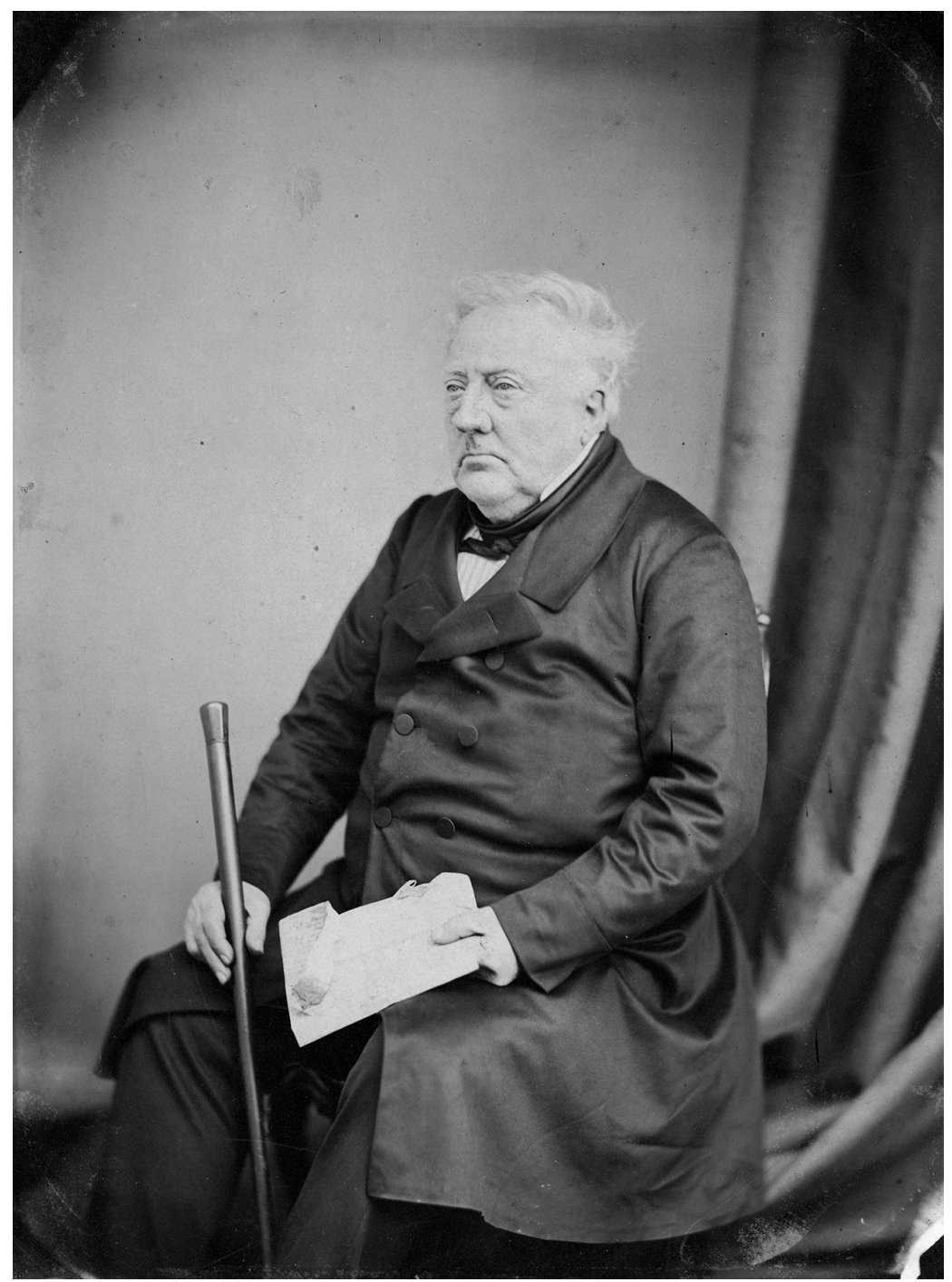

3. Cet article se situe dans le droit fil d'un master soutenu en 2013 à l'université de Nantes. Voir : DANIEL, Jacques, Louis-Jacques-Marie Bizeul : l'archéologue. Une contribution à l'histoire de l'archéologie dans l'Ouest armoricain, Bertrand JolY et Martial MonTEIL (dir.), mémoire de master, université de Nantes, 2013. 


\section{J. M. Bizeul : sa trajectoire. De l'ancrage local d'un notable de province à la stature revendiquée d'un homme de science}

En ce début du mois d'octobre, L. J. M. Bizeul est entré dans sa soixantième année et cependant sa notoriété est toute récente. Elle remonte aux années 1841 et 1842, lorsque, pour répondre aux pressantes sollicitations de son ami F. M. Cayot-Délandre, il avait fait paraître dans l'Annuaire $d u$ Morbihan une étude sur les voies romaines de Bretagne ${ }^{4}$. Elle l'avait imposé comme le spécialiste reconnu d'un domaine demeuré, avant lui, marginal. Entre autres résultats, la publication eut celui de retenir l'attention d'Arcisse de Caumont. Le célèbre antiquaire normand lui ouvrit alors les colonnes du Bulletin monumental en même temps que les portes de sa Société française pour la conservation des monuments nationaux. Au mois de juin 1843, à la veille du congrès de Vannes où se décida la création de l'Association bretonne, il lui décerna une médaille d'argent pour ses travaux et le fit admettre comme membre titulaire de l'Institut des provinces. Lors de ce congrès, L. J. M. Bizeul, à ses côtés, œuvra victorieusement pour que le projet initial de l'association, voué primitivement à la seule modernisation de l'agriculture, intègre également l'archéologie ${ }^{5}$.

Cependant, avant d'accéder à la notoriété, son parcours fut plutôt laborieux tant il est vrai que pour un savant de ce type, qualifié, faute de mieux, d'amateur, la quête scientifique a dû sans cesse composer avec l'exercice de l'activité professionnelle, de la chose publique et de la vie familiale $^{6}$. À lire son journal et sa correspondance, rien, dans les années de jeunesse de L. J. M. Bizeul, ne laissait présager sa future carrière d'érudit et d'archéologue. On le voit surtout s'employer, à l'exemple de son père,

4. BizEul, Louis Jacques Marie, "Des voies romaines de la Bretagne et en particulier de celles du Morbihan ", Annuaire du Morbihan, 1841, p. 115-262 et leur "supplément " Annuaire du Morbihan, 1842, p. 1-22.

5. Sur la fondation de l'Association bretonne, voir GuIOMAR, Jean-Yves, Le bretonisme. Les historiens bretons au XIXe siècle, Mayenne, 1987, p. 131-149.

6. Les développements qui suivent sont, pour la plus grande part, redevables aux importants fonds d'archives déposés par la famille de Louis Jacques Marie Bizeul, à la médiathèque de Nantes pour l'un, aux Archives départementales de Loire-Atlantique pour l'autre. Le fonds de la médiathèque est composé essentiellement de manuscrits, pour beaucoup inédits, et de quelques papiers personnels. Celui des Archives départementales de Loire-Atlantique est classé sous la cote 2 J. Son « journal " (2 J 4), comprend un Mémorandum, sorte de carnet qui (deux annotations concernant les années 1803 et 1804 mises à part) couvre les années 1805 à 1813 et un second ensemble très différent (18381841 puis 1844-1861) constitué de cahiers dont la fonction principale semble avoir été de noter les dépenses occasionnées par ses déplacements. C'est si vrai que les parties manquantes de cet ensemble (1832-1837 et 1841-1844) sont classées en 2 J 5 (comptabilité : notes, cahier de comptes). Pour des références plus générales, au toujours utile CHALINE, Jean-Pierre, Sociabilité et érudition. Les sociétés savantes en France, Paris, CTHS, 1995, on ajoutera : PARSIS-BARUBE, Odile, La province antiquaire. L'invention de l'histoire locale en France (1800-1870), Paris, CTHS Histoire, 2011; Ploux, François, Une mémoire de papier. Les historiens de village et le culte des petites patries rurales, PUR, 2011; HARRISON, Carol E, The bourgeois citizen in nineteenth-century France, Oxford, Oxford university press, 1999; JoHnson, Christopher H, Becoming bourgeois : Love, kinship and power in provincial France (1670-1880), London, Cornell university press, 2015. 
à occuper une place au sein de la bourgeoisie capacitaire et soigner son ancrage local, puisque l'état de la fortune familiale ne lui permet pas de vivre de ses rentes.

\section{Un digne représentant de la bourgeoisie capacitaire ${ }^{7}$}

Figure 2 - Médaille décernée par A. de Caumont, 1843. “Voies romaines de Bretagne. M. Biseul »(Musée de Blain)

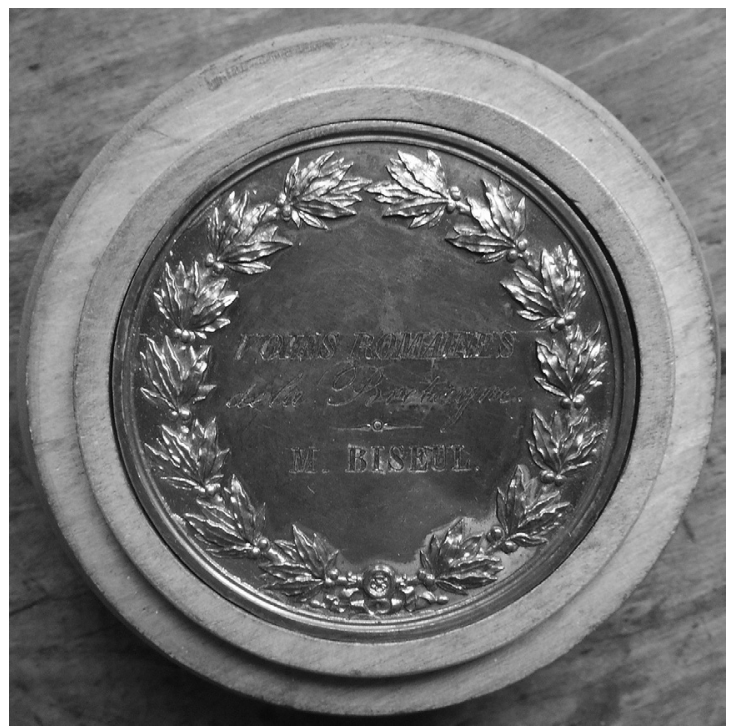

Jacques Bizeul (1749-1813), le père, issu d'une famille de paysans aisés dont on peut suivre la trace à Blain (Loire-Atlantique) depuis le milieu du $\mathrm{XVII}^{\mathrm{e}}$ siècle, dut en grande partie son ascension sociale à la protection accordée par le duc de Rohan. Louis Marie Bretagne Dominique de Rohan-Chabot le fit en effet notaire et greffier de son marquisat de Blain avant qu'il ne cumule ces charges, en 1779, avec celle de notaire royal. En 1791, depuis Nice où il s'était réfugié, il le désigna en outre comme son archiviste, chargé de veiller à la bonne conservation de son chartrier, avec comme corollaire l'obligation de résider en son château de la Groulais.

C'est peu dire que la période révolutionnaire éprouva durement sa fidélité à la maison de Rohan. Son loyalisme le retint de participer à la rédaction du cahier de doléances et conduisit ce royaliste modéré de conviction à faire partie, sur la scène politique blinoise, du camp des " aristocrates ",

7. Pour d'utiles comparaisons avec la trajectoire de Louis-Jacques-Marie Bizeul, on consultera avec profit Johnson, Christopher H, Becoming bourgeois..., op. cit. Le chapitre 10, notamment, concerne Jean-Marie Galles, imprimeur, un des fondateurs de la Société polymathique du Morbihan, conservateur du musée de 1828 à 1860 et son fils Louis (1827-1874), archéologue et imprimeur-libraire à Vannes. 
ainsi dénommé par ses adversaires ${ }^{8}$. Et c'est de manière assez complexe qu'il parvint, sous la Terreur, à obtenir un certificat de civisme et, par là même, à conserver son étude notariale. Finalement, la consécration vint du Consulat et de l'Empire : Napoléon Bonaparte fit en effet de cet homme de loi, propriétaire foncier important, un notable ${ }^{9}$.

De son union avec Marie Gautier naquirent quatre enfants, dont deux moururent en bas âge. L'aînée, Marie (1778-1864), épousa Pierre Gergaud, un épicier installé à Nantes, 21 rue de la Juiverie. Aussi, c'est sur le dernier-né, Louis Jacques Marie (1785-1861), qu'il reporta tout son désir de perpétuer l'ascension sociale familiale, lui donnant une éducation propre à intégrer l'élite administrative de son temps. Dans ce but, L. J. M. Bizeul acquit, à la pension Rouzic à Nantes ${ }^{10}$, une solide formation secondaire, nourrie d'humanités classiques, qu'il perfectionna ensuite en intégrant, en l'an X, la classe de grammaire et de belles-lettres de l'École centrale. Non dénué de fantaisie ${ }^{11}$, il s'y révéla un élève particulièrement brillant qui obtient, notamment, le premier prix de rhétorique ${ }^{12}$. Le thème en était le suivant : " De ces trois qualités, mémoire, sensibilité, justesse d'esprit, quelle est la plus nécessaire à l'homme de lettres? "Au terme d'un discours de quelque 25 pages, il concluait de la manière suivante : " [...] tout homme qui réunira une imagination vive et brûlante, une sensibilité exquise et qui saura les diriger par la justesse d'esprit produira alors ce bel ensemble que si peu d'auteurs ont pu trouver et qui peut seul procurer une gloire solide et durable ${ }^{13}$ ". Par-delà le caractère convenu de l'exercice, on voit assez clairement se dessiner chez L. J. M. Bizeul, dès ce moment, une nette ambition intellectuelle.

De son côté, Jacques, le père, prodiguait des encouragements. Il veilla même à lui donner une formation pratique en l'initiant aux méthodes de l'arpentage, technique fort utile dans l'exercice du métier de notaire. Car son projet était bien de transmettre son étude à son fils. À charge, pour celui-ci, d'acquérir un bon bagage en droit.

"Ce (degue) ca (dga) hier (deguier) a (dgar) pa (dga) rti (dgui) yen (dguen) a (dga) moi (dguoi) Bi (dgui) (dgui)zeu (dgueu) le (degue) é (dgué) tu (dgû) di (dgui) an (dgan) a (dga) la (dga) pen (deguen) sion (degon) rou (dgou) zi (degui) que (dgue) a (dga) nan (dgan) tes (degue) ru (dgu) de (dgue) Sul (dgul) ly (dgui) nu (dgu) mer (dguer) ro (dgo) 4 ".

8. Maire de Blain en décembre 1792, Jacques Bizeul fut destitué après l'insurrection de mars 1793 où il avait pourtant été pris à partie. En novembre 1793, il tenta, vainement, de s'opposer à la destruction par les flammes du chartrier dont il avait la garde.

9. Il siège au conseil général de l'an VIII à 1810 aux côtés de Pierre Louis Athénas et accède à la fonction de maire-adjoint de Blain qu'il exerce jusqu'à son décès en 1813.

10. La pension Rouzic, 4 rue de Sully, est l'une des six écoles secondaires de Nantes. En l'an XI, elle compte quatre professeurs

11. Il fonde, avec quelques amis, une Société gastronomique dont les statuts, mi-parodiques, mi-sérieux, miment, dans un esprit potache, ceux d'une organisation secrète.

12. Le jury, présidé par le préfet Letourneur, comprend Richard jeune, Pierre Louis Athénas et du Bochet.

13. Archives du musée de Blain, carton Bizeul. 
Figure 3 - Premières pages du cahier de Géographie de L. J. M. Bizeul, pension Rouzic, Nantes (Musée de Blain)

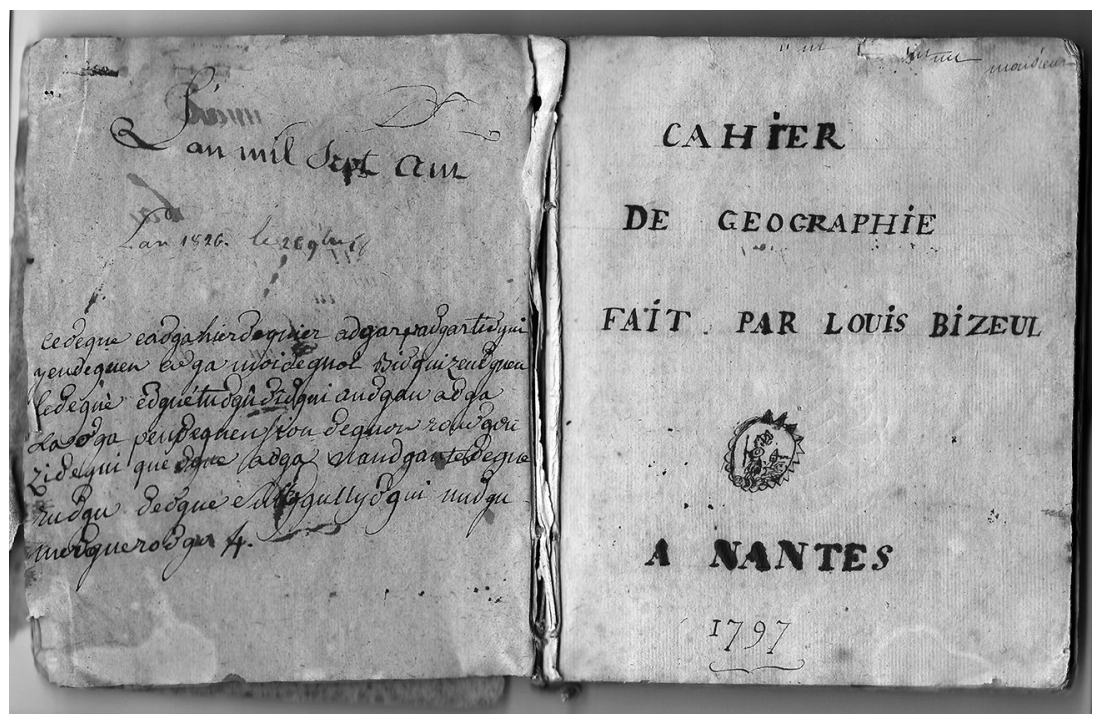

Une fois levée l'épée de Damoclès du service militaire ${ }^{14}$, L. J. M. Bizeul s'inscrivit donc, en 1806, à l'école de droit de Rennes. Après le baccalauréat obtenu en juillet $1808^{15}$, il poursuivit son parcours par une licence (droit romain en latin et droit français) brillamment acquise l'année suivante. Au terme de ce cursus, il décida de s'établir comme avocat. Cependant, L. J. M. Bizeul souffrait d'un défaut rédhibitoire pour l'exercice de cette profession : il était bègue. Aussi, après avoir vainement tenté d'obtenir un poste à Paris, au ministère de la justice, se résolut-il à accepter l'idée d'être notaire à Blain. Comme le dit sobrement l'auteur d'un brouillon de notice nécrologique retrouvé dans les papiers de la famille ${ }^{16}$ : «Reçu avocat [...] il quitta Rennes et vint à Blain succéder à son père dans les fonctions de notaire toute autre carrière qui eût été plus à son goût lui étant fermée par la très grande difficulté qu'il avait à s'exprimer. "

Pourtant, cette solution de repli faillit ne pas réussir. La loi du 17 mars 1803 réformant la pratique du notariat instaurait, notamment, un numerus clausus. Et il y avait, à Blain, une étude de trop. Le retrait de Jacques Bizeul n'aurait donc conduit qu'à la suppression pure et simple de son étude. Seul un hasard providentiel permit la réalisation de ce plan : le décès subit, le

14. Le 2 nivôse, an XIV, il est définitivement dispensé, car reconnu " incapable de supporter les fatigues de la guerre pour cause d'infirmité ". Une décision de complaisance due vraisemblablement à l'intervention de Jacques Bizeul.

15. Louis Jacques Marie Bizeul obtint la première partie le 1/06/1807 et la seconde le 19/07/1808.

16. Arch. dép. de Loire-Atlantique, $2 \mathrm{~J} 3$. Notice probablement rédigée par son fils Raymond (1818-1884) chez lequel il passa la fin de sa vie. 
27 septembre 1810, vers quatre heures du matin, sous les halles de Blain, du notaire Jean-René Paul Boudier. Le décret de nomination de L. J. M. Bizeul fut signé le 13 décembre. Le 7 janvier 1811, il prêta serment devant le tribunal de grande instance de Nantes. Le lendemain, il commença à emménager avec son épouse et son jeune fils et put écrire dans son journal «nous avons commencé à manger à notre part ${ }^{17}$ ".

L'incidence qu'eut la profession notariale sur la carrière érudite de L. J. M. Bizeul a été diversement appréciée par ses contemporains. Prosper Levot, qui le fréquenta pendant de longues années, souligne la gêne qu'elle occasionna pour ses recherches ${ }^{18}$. Arthur Lemoyne de La Borderie, qui le connut moins, écrit, dans une formule ambiguë, qu'il " embrassa " la carrière notariale et qu'elle contribua à développer " ce goût de la recherche historique qui, pour le fils du dernier archiviste des Rohan, était d'ailleurs comme une part de l'héritage paternel ${ }^{19}$ ". La réalité fut toute autre.

\section{J. M. Bizeul et le notariat : une affaire de famille?}

À peine son baccalauréat en poche, L. J. M. Bizeul avait épousé à Nantes, le 30 août 1808, Léonice Hérault (1781-1846), fille et petite-fille de notaires nantais, de quatre ans plus âgée que lui. De notaire, Jean-Baptiste Pierre, le père de Léonice, décédé en 1803, n'avait conservé que le titre ${ }^{20}$. Quant à Louise Françoise Ricard, sa mère, elle était morte deux ans après sa naissance. Orpheline, Léonice avait toutefois quelque famille à Rennes. Une de ses tantes y était installée avec son époux Jean-Baptiste Joseph Barbarin, un pharmacien réputé. Louise Barbarin, enfant du couple et cousine, donc, de Léonice y était l'épouse de Pierre Lesbaupin, une personnalité fort estimée comme avocat, bâtonnier de l'ordre et, ce qui ne pouvait qu'intéresser L. J. M. Bizeul, professeur à l'école de droit ${ }^{21}$.

Les deux jeunes gens s'étaient rencontrés pour la première fois le 21 février $1806^{22}$. Toutefois, leur idylle, ponctuée par un "O love " dans son journal, ne commença véritablement que le 26 mai suivant, soit quatre jours, précisément, avant son départ pour Rennes. Les convenances furent scrupuleusement respectées. Dans l'attente d'obtenir une situation, L. J. M. Bizeul fit sa cour à Léonice Hérault pendant plus de deux années. Les promis se rencontraient à l'occasion des vacances scolaires tandis qu'à

17. Arch. dép. de Loire-Atlantique, $2 \mathrm{~J} 4$.

18. LeVot, Prosper, " Notice nécrologique sur M. L. J. M. Bizeul de Blain ", Bulletin de la Société archéologique de Brest, t. 3, 1862-1863, p. 261-270.

19. La Borderie, Arthur Lemoyne DE, "Chronique ", Revue de Bretagne et de Vendée, t. 9, $5^{\mathrm{e}}$ année, 1861, p. 331.

20. Le certificat de civisme lui ayant été refusé, son étude fut "suspendue "sous la Terreur. En l'absence de démarches de sa part, la mesure ne fut jamais rapportée.

21. À ce titre, Pierre Lesbaupin fera partie des jurys qui délivreront le baccalauréat et la licence de droit, brillamment obtenus par Louis Jacques Marie Bizeul.

22. Arch. dép. de Loire-Atlantique, 2 J 4. Ce jour-là, Louis Jacques Marie Bizeul note dans son journal : "I have seen for the first time miss $L^{c e} h$ ". Mais il s'agit sans doute d'un ajout postérieur. 
Rennes, il travaillait avec succès à conquérir l'estime des familles Barbarin et Lesbaupin. La décision du mariage fut arrêtée lors du séjour que Léonice effectua chez sa tante Barbarin pendant les mois de février à mai 1808. Un mariage qui, chaleureusement approuvé par Jacques Bizeul, n'eut, contrairement aux apparences, rien d'arrangé.

Sans doute, l'alliance matrimoniale contractée par L. J. M. Bizeul allaitelle dans le sens de ses intérêts de carrière. On ne peut douter cependant de sa vive attirance pour Léonice. Si, dans son journal, avant son mariage, il évoque leurs rencontres par des notations telles que " a very beautiful day " alternant avec des " a very fine day ${ }^{23}$ ", après la cérémonie, le ton change : elles se muent en " a very good night " accompagnées, toujours en anglais, du nombre de rapports sexuels qu'il a avec sa jeune épouse. Leur durée et leur fréquence, inscrite sur une échelle de un à trois, voire trois et demi, révèlent chez lui une vigueur sexuelle insoupçonnée jusqu'alors ${ }^{24}$. Le regard posé sur l'intimité du couple qu'autorise son journal montre donc, à l'évidence, que l'union entre L. J. M. Bizeul et L. Hérault a scellé une vraie rencontre amoureuse. Cependant, quoique vive, l'attirance des deux jeunes gens est restée parfaitement compatible avec les convenances affichées par la société de cette époque. Leur vie familiale ne s'écartera pas de ce modèle.

Une lettre de Léonice à son frère Sévère ${ }^{25}$ donne à voir leur genre de vie au début de la Restauration. Tous les matins, elle se lève à cinq heures et demie puis, après avoir donné des ordres aux domestiques, va à la messe. Le reste de la matinée est consacré à l'éducation de ses enfants. " Après avoir fait cela ", écrit-elle, " je vais au cabinet de mon mari et y travaille ordinairement jusque à 1 heure. Il n'a pas d'autre clerc que moi, je fais toutes ses grosses et une partie de ses minutes ce qui me flatte infiniment ». Et d'ajouter : « Il a par continuation l'estime générale de tous ceux qui le connaissent. Il jouit de la meilleure considération, il n'a d'autre défaut que celui d'être vif mais je sais le prendre et il n'est fâché qu'autant que je le veux bien. " Après le repas, elle coud ou tricote et ne " donne jamais de temps à la lecture d'aucun livre " car, dit-elle, " mon genre d'amour-propre est de ne vouloir rien leur devoir et de rester avec ce que j'ai reçu de la nature ".

La famille mène une existence somme toute bien réglée, agrémentée par les nombreuses soirées dansantes organisées chez les membres de la bonne société blinoise et qu'animent une douzaine de jeunes gens, " tous d'un bon ton et de bonne société ", venus là pour le creusement du canal de Nantes à Brest. De cette époque quelque peu mondaine, il nous reste une petite pièce, "La fête du village ou le mariage de Caroline ", que

23. Arch. dép. de Loire-Atlantique, $2 \mathrm{~J}$ 4. On relève 100 occurrences dans son journal. Lorsqu'il évoque ses rencontres avec Léonice, c'est au moyen de phrases stéréotypées exclusivement rédigées en anglais.

24. Alain Corbin a attiré l'attention sur cette "étonnante arithmétique coïtale " dont font aussi preuve Flaubert, Michelet, Victor Hugo, les Goncourt... (CoRBIN, Alain, "L'arithmétique des jours au XIXe siècle ", Traverses, 1985; repris dans Le temps, le désir et l'horreur, essais sur le XIXe siècle, Aubier, 1991 ; réédité en coll. " champs histoire ", Flammarion, 1998 et 2014, p. 18-19 de la dernière édition).

25. Arch. dép. de Loire-Atlantique, 2 J 21, Lettre du 24 avril 1814. 
L. J. M. Bizeul commit avec A. J. Cottin de Melleville en $1816^{26}$. Cependant, une fois les travaux interrompus, la petite ville va retomber dans une sorte de léthargie. On en relève la trace, comme en écho, dans ce passage écrit par L. J. M. Bizeul à son beau-frère, où l'on sent poindre un certain ennui : "Retiré dans une petite ville qui peut être regardée comme campagne, une bibliothèque est un meuble fort utile ${ }^{27}$. "Une discordance, sans doute, avec les aspirations de Léonice...

Le couple aura quatre enfants. Après Louis (1809-1844), naîtront Sévère (1811-1878), Perrine (1817-1833) et Raimond, (1818-1884). À l'exception de Perrine, décédée à l'âge de 16 ans, tous feront des études supérieures et accéderont à des professions libérales. Sévère mis à part ${ }^{28}$, les autres enfants perpétueront l'étude paternelle, donnant ainsi l'image, quelque peu illusoire, d'une continuité familiale et professionnelle voulue et assumée ${ }^{29}$. Notaire par défaut, L. J. M. Bizeul s'est en effet empressé de céder son étude dès que ses enfants ont été en capacité de gagner leur vie ${ }^{30}$. Finalement, quoiqu'exercée consciencieusement, cette profession n'aura été pour lui qu'une activité alimentaire qui lui aura permis, tout au plus, d'asseoir une certaine notoriété. Le jugement porté par son fils Raimond, probable auteur du brouillon de notice nécrologique déjà évoqué, est sans ambiguïté : " Mais l'amour de la science s'était déjà emparé de lui; aussi sa longue carrière notariale ne fut-elle point une source de fortune pour lui. "

Sans doute faut-il voir, dans cet " amour de la science ", l'origine de sa tentative, infructueuse mais menée avec constance entre 1824 et 1829 , pour récupérer l'étude nantaise de son beau-père. Loin de témoigner d'une inclination particulière pour cette profession, il faut plutôt la lire comme une occasion, pour lui, de quitter cette " petite ville qui peut être regardée comme campagne ", de se rapprocher de ce foyer culturel nantais qu'il désirait pleinement intégrer depuis son admission, en 1821, comme membre correspondant de la Société académique de Nantes (cf. infra). D'ailleurs, au cours de cette période, L. J. M. Bizeul songea également à devenir magistrat, juge de paix ou bibliothécaire dans une grande ville, Nantes de préférence ${ }^{31}$. La révolution de Juillet et la perte de ses appuis politiques sonnèrent le glas de ses espoirs devenus désormais quelque peu chimériques. Il se résolut finalement à lier son existence à la petite ville de Blain. Son riche passé,

26. Bizeul, Louis Jacques Marie, CotTin de Melleville, A. J., La fête du village ou le mariage de Caroline, Vaudeville en un acte, joué à Blain, à l'occasion du mariage du duc de Berry, Médiathèque de Nantes, ms 753, 22 feuillets in $4^{\circ}$.

27. Arch. dép. de Loire-Atlantique, 2 J 21, Lettre du 16 septembre 1817.

28. Sévère passe sa thèse de médecine à Paris en 1836. Il fut médecin à Nantes puis à Blain.

29. Louis Jacques Marie Bizeul cédera son étude à Louis en 1835 et, après le décès accidentel de celui-ci en 1844, à Raimond, son dernier-né. Louis, le seul sans doute à s'intéresser vraiment à la carrière de notaire, décéda à la suite d'un accident de diligence alors qu'il revenait de Nantes après avoir négocié à son profit le rachat de l'étude du notaire du Pont-du-Cens. Quant à Raimond, il abandonna le notariat en 1854 pour devenir juge de paix.

30. Arch. dép. de Loire-Atlantique, 2 J 9, La cession de l'étude à Louis en 1835 était fictive. Elle sera effective en 1837 à la veille du mariage de Sévère.

31. Arch. dép. de Loire-Atlantique, 2 J 8, lettre du 8 mars 1826. 
qu'il entreprit alors d'étudier, devait, à ses yeux, en rehausser l'éclat. Nous y reviendrons. Mais avant d'en venir à son activité dans ce domaine, il convient de parachever à grands traits le portrait de notre érudit...

\section{J. M. Bizeul et la terre : les racines campagnardes}

Bizeul a conservé, d'une enfance et d'une adolescence marquées par la vie rurale, un genre de vie campagnard. Il note en tête de son journal : "L'an 1803, notre vigne a produit une barrique de vin. L'an 1804, elle a produit huit barriques de vin et nos vergers de la Tourotais trois barriques de cidre. " L. J. M. Bizeul sait monter à cheval. Il chasse. En 1801, la préfecture lui délivre un " permis de porter un fusil [...] pour chasser sur ses terres et celles d'autrui [...] et à porter en voyage un sabre et des pistolets ${ }^{32}$ ". C'est en outre un excellent marcheur. À l'exception de Nantes et de Rennes, son horizon se limite au milieu rural proche et, contrairement à beaucoup d'antiquaires de sa génération, on ne lui connaîtra aucun voyage à l'étranger. Le 28 novembre 1805, à Bourgneuf, - il a alors vingt ans - il écrit avec toute la précision que requiert un tel événement : " J'ai vu la mer pour la première fois à cinq heures moins 10 minutes du soir ${ }^{33}$. "

On retrouve ce tropisme campagnard, idéalisé, dans "La solitude », un texte de sa composition qu'il lut à l'École centrale le 3 juin $1802^{34}$ :
"Oui, c'est loin des villes, c'est au milieu des champs
Qu'on trouve des plaisirs si simples et touchants;
C'est sur l'émail fleuri d'une longue prairie
Que l'âme vient reprendre une active énergie
C'est à l'ombre des bois, c'est au bord des ruisseaux
Que le cœur se remplit de sentiments nouveaux
C'est à l'abri sacré de ces chênes antiques
Qu'on se sent agités de transports poétiques!
Et que l'esprit, des cieux atteignant la hauteur,
S'élève sur les vaux et vole à son auteur "

Plus prosaïquement, L. J. M. Bizeul fut aussi un propriétaire foncier, statut indissociable de la notabilité à cette époque. Peu après le décès de son père survenu en 1813 , il hérita de la moitié du domaine paternel à la suite d'une donation partage que sa mère effectua. Augmenté des terres apportées par L. Hérault et de quelques acquisitions, ce patrimoine, bien que consistant, ne fit toutefois pas de lui un grand propriétaire ${ }^{35}$. Tel quel, il n'aurait pas suffi à subvenir à ses besoins et à ceux de sa famille. Il lui procura cependant un appréciable revenu d'appoint qui lui permit de vivre dans une confortable aisance.

32. Arch. dép. de Loire-Atlantique, 2 J 3.

33. Arch. dép. de Loire-Atlantique, $2 \mathrm{~J} 4$, Mémorandum.

34. Archives du musée de Blain. Carton Bizeul.

35. Arch. dép. de Loire-Atlantique, 2 J 3 et 2 J 14. En 1845, Louis Jacques Marie Bizeul possède quatre habitations simples et quatre exploitations rurales (La Pironnerie, la Ridelais, le grand moulin, la Chèvrerie) qu'il afferme. La plus importante (La Pironnerie, 41 ha 04 a 11 ca en labours, près et landes) a été apportée par Léonice Hérault. 
L. J. M. Bizeul et son " Aperçu général sur l'étude des voies romaines "

Figure 4-Permis de porter un fusil

(Archives départementales de Loire-Atlantique, 2 J 3)

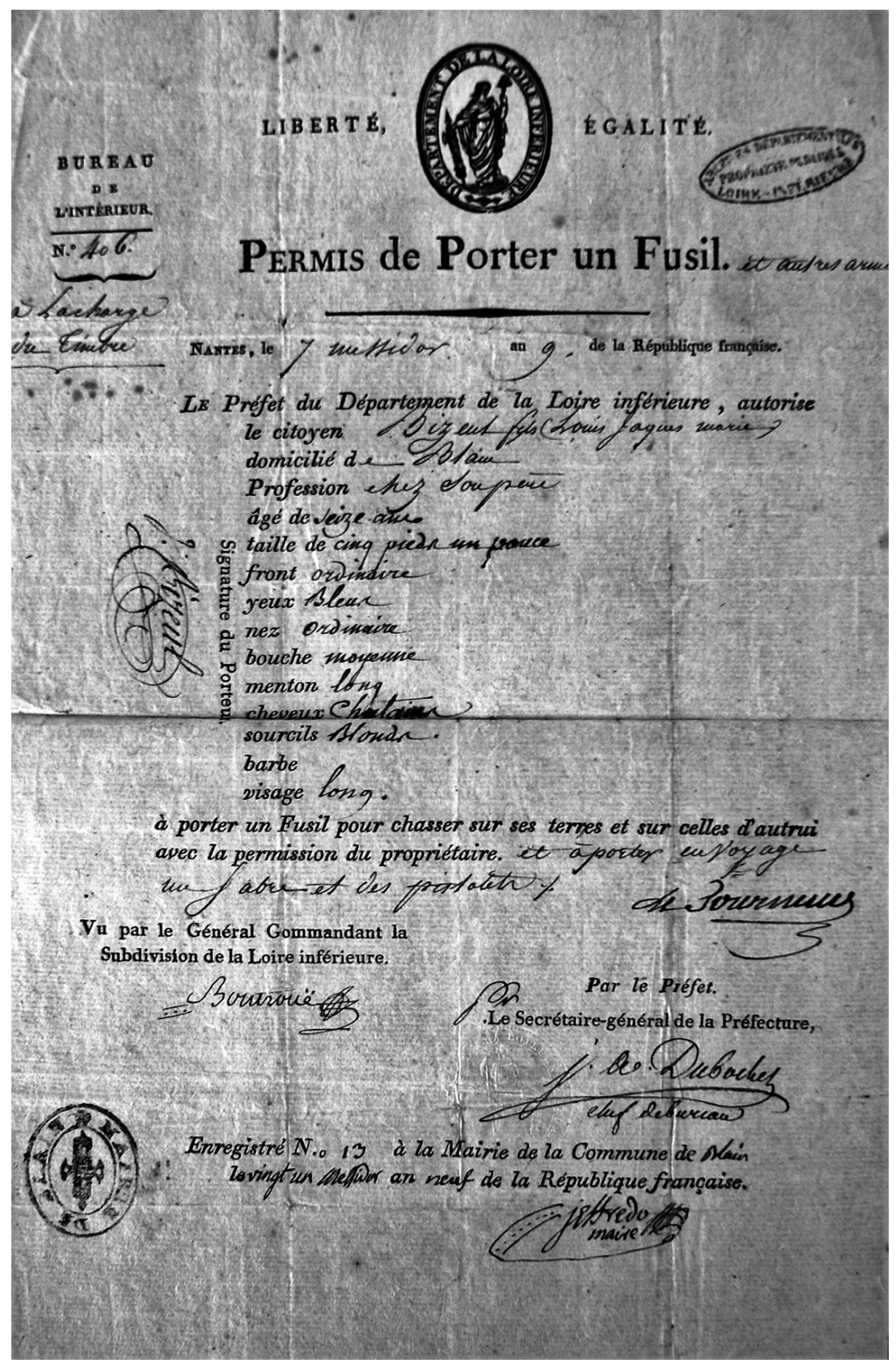


Ses archives personnelles le montrent attentif à la gestion de son domaine, qu'il afferme en totalité. À titre d'exemple, le contrat de 9 ans qu'il passe en 1844 avec les frères Lemaitre pour l'exploitation de la Pironnerie prévoyait un loyer annuel de 1000 francs, plus $20 \mathrm{~kg}$ de "bon beurre ", 20 poulets " bons à manger ", 20 paquets ordinaires de filasse, $1500 \mathrm{~kg}$ de paille, 18 décalitres de blé noir, 9 décalitres de pommes de terre, un morceau de lard d'au moins $1,5 \mathrm{~kg}$ à chaque abattage de cochon ${ }^{36}$. Il n'hésite pas, face à un mauvais payeur, à porter l'affaire devant les tribunaux. Ainsi, en 1835 fait-il condamner la veuve Prétral, pourtant insolvable, à la saisie de ses biens, dont une vache et une génisse qu'il fait amener à Blain pour être vendues à la foire du Gâvre.

L. J. M. Bizeul a-t-il été un propriétaire éclairé? Il est en tout cas très au fait des problématiques concernant la modernisation de l'agriculture. Aux yeux de l'administration, il fait même figure d'expert, ce qui lui valut de siéger, en 1829, au comité consultatif d'agriculture pour l'arrondissement de Savenay. Il y a pour mission de donner un avis sur " les projets et mémoires relatifs à l'agriculture et de présenter ses vues sur les améliorations et perfectionnements qui pourraient contribuer aux progrès de l'agriculture et sur les encouragements et récompenses à accorder ${ }^{37}$ ". Il s'y montre très assidu, comme il le sera aussi aux séances agricoles de l'Association bretonne $^{38}$. Son président, Jules Rieffel, qu'il connaît bien, avait fondé, en 1830, sur le domaine de Grand-Jouan dans la commune voisine de Nozay, une école expérimentale d'agriculture unique en France, à partir du défrichement de 500 ha de landes. Un exemple imité par L.-J.-M. Bizeul en 1836 lorsqu'il fit enclore, pour la mettre en valeur, une certaine quantité de terrains dans la lande de Bec-la-Haye, près de la forêt du Gâvre, ce qui, dit-il, devait " puissamment favoriser l'agriculture dans la commune de Blain ${ }^{39}$ ".

C'est à la croisée de son expérience de propriétaire et de ses compétences de notaire qu'il convient de mentionner son activité de régisseur. On le voit par exemple, à Bouvron, s'occuper des domaines d'Amaury Marie de Fourché, comte de Quehillac et de son beau-frère Jean-Marie Walsh, vicomte de Serrant. Mais c'est surtout auprès de Louis du Cambout, quatrième marquis de Coislin (1769-1837), qui tenait résidence en son château de Carheil dans la commune voisine de Plessé, qu'il consacra le plus de temps - 15 à 20 ans selon ses dires - à cette fonction. On ne saurait en sous-estimer l'importance. Car, outre les 1350 ha constituant le domaine de Carheil, le marquis possédait des terres en basse Bretagne et, de plus, il lui arrivait fréquemment de résider à Paris. Tout cela conduisit L. J. M. Bizeul à effectuer, le plus souvent en diligence, de longs déplacements qu'il tenta de mettre à profit pour explorer des dépôts d'archives ou suivre la trace de voies romaines.

36. Arch. dép. de Loire-Atlantique, 2 J 14

37. Arch. dép. de Loire-Atlantique, $2 \mathrm{~J} 3$

38. En 1845, au congrès de Nantes, il rédige un mémoire de 14 pages intitulé " Des bassins calcaires de la Loire-inférieure et en particulier de celui de Campbon et de saintGildas ", publié dans L'agriculture de l'Ouest, la revue dirigée par J. Rieffel.

39. Arch. dép. de Loire-Atlantique, $2 \mathrm{~J} 14$. 


\section{Une fidélité royaliste à l'épreuve}

En s'installant à Blain, L. J. M. Bizeul était promis à une carrière de notable au service de l'Empire, dans la continuité de celle qu'exerçait son père. Après son décès survenu en 1813, c'est tout naturellement qu'il lui succéda dans la fonction d'adjoint au maire de Blain. Il y avait été bien préparé et, sans doute, cette perspective n'était-elle pas pour lui déplaire.

Jeune étudiant à l'école de droit de Rennes, déjà, il avait été désigné pour participer à la garde d'honneur à pied qui devait accueillir Napoléon lors de sa tournée de l'été 1808 dans les départements de l'Ouest. La rencontre annoncée eut lieu le 11 août, à Nantes, où L. J. M. Bizeul se trouvait en vacances. Il approcha l'Empereur pendant dix minutes. De lui, il retint qu'il " était vêtu très simplement " et que " son air était gracieux et affable ". Un commentaire somme toute assez neutre qui contraste avec le claironnant "Vive le roi » qu'il écrivit dans son journal lors de l'avènement de Louis XVIII et que redoubla un "Vive le roi quand même " à l'annonce de l'entrée de Napoléon à Paris au début des Cent Jours. Commencée sous l'Empire, sa carrière politique, quoique limitée à l'échelon local, connût une nette accélération après l'échec de l'ultime retour de «l'usurpateur »; il entra au conseil d'arrondissement de Savenay puis accéda à la fonction de maire de Blain ${ }^{40}$. Mais, à peine amorcée, elle vint se briser sur la révolution de juillet 1830 : à l'instar de beaucoup de notables de l'Ouest, comme lui légitimistes, il préféra démissionner plutôt que de prêter serment à Louis Philippe ${ }^{41}$.

L. J. M. Bizeul fut donc un royaliste proche des milieux ultra. La tradition familiale l'y disposait. À cela s'ajoutèrent les traumatismes nés de la guerre civile. Encore enfant, ne se trouvait-il pas au château de La Groulais lorsque, à l'été 1793, la garnison profana l'enfeu de la famille de Rohan et dispersa les restes des défunts dans la cour ${ }^{42}$ ? Et, surtout, ne fut-il pas le témoin de la destruction par les flammes, au mois de novembre 1793, du chartrier des Rohan dont son père, archiviste, avait la garde ${ }^{43}$ ? Si l'on en croit A. L. de La Borderie, soutenu sur ce point par P. Levot, plus d'une fois il en avait conté la destruction " avec émotion, presque avec larmes ${ }^{44}$ ". Quelques cartons ayant échappé à l'autodafé, il craignit, lorsque survint la révolution de juillet 1830, qu'ils ne subissent le même sort. Prenant alors à témoin Miorsec de Kerdanet, l'érudit de Lesneven, il écrivit : " [ces événements] sont venus me faire craindre de voir, comme mon pauvre père, la dernière destruction

40. Louis Jacques Marie Bizeul est nommé conseiller d'arrondissement le 11/04/1821, maire de Blain au mois d'août 1825 .

41. Arch. dép. de Loire-Atlantique, 3 M 294. Le 25 novembre 1839, il fut élu au conseil d'arrondissement contre le maire de Blain, celui-là même qui l'avait remplacé après sa démission. Il prêta serment. Le conseil d'arrondissement n'avait qu'un rôle consultatif.

42. LEgouAIS, Francis, Des mâcles des Rohan au bonnet républicain, Nantes, 1974 p. 159.

43. du Halgouët, Henri, "Le chartrier de Blain ", Annales de Bretagne, t. 35, n 1, 1921. p. 81-88. Ses 80 volumes avec inventaire et table analytique occupaient deux étages dans une des tours du château.

44. LA Borderie, Arthur LEMOYNE DE, "Chronique ", Revue de Bretagne et de Vendée, t. 9, $5^{\mathrm{e}}$ année, 1861, p. 331 . 
de ces vieux patrimoines qui ont traversé les siècles et les guerres pour devenir la proie des nouveaux révolutionnaires ${ }^{45}$ ".

Le sort fait aux archives de la maison des Rohan-Chabot contribua sans nul doute à nourrir chez L. J. M. Bizeul une profonde détestation du vandalisme révolutionnaire; en contrepoint, il permet d'éclairer l'influence considérable qu'exerça sur lui le marquis de Coislin, éminent représentant du royalisme ultra en Loire-Inférieure ${ }^{46}$.

Le brouillon de notice nécrologique ${ }^{47}$ nous apprend qu'au début de sa carrière notariale, devenu régisseur de la maison de Coislin, il " trouva dans les nombreuses archives de cette famille un aliment qui développa au plus haut degré son goût pour les choses du temps passé et absorba jusqu'à sa vie ". L'intérêt exceptionnel que porta L. J. M. Bizeul à ces archives ne tint pas uniquement à leur fonction documentaire. Elles furent, en effet, promises à la destruction, mais le temps manqua aux révolutionnaires pour mettre leur projet à exécution : rescapées à ses yeux du vandalisme révolutionnaire, elles revêtirent une charge émotionnelle forte qui vint rappeler la perte irrémédiable des archives dont son père avait eu la garde. De ce point de vue, on peut dire que la relation entre L. J. M. Bizeul et le marquis de Coislin fut presque d'ordre filial. Quoi qu'il en soit, son attachement au marquis et à sa famille ne se démentit pas. Le 9 juillet 1837, ils furent seulement trois, dont L. J. M. Bizeul, à veiller toute la nuit, hommage ultime, le corps du défunt marquis ${ }^{48}$. C'est à lui que fut confié le soin de rédiger son éloge funèbre ${ }^{49}$. Lui encore qui représenta les intérêts de la marquise lors du partage des biens. Lui enfin que la marquise douairière désigna comme son fondé de pouvoir à l'occasion de la vente du château de Carheil au prince de Joinville, au mois d'octobre 1842.

Replacée dans le climat intellectuel de la Restauration, où le préambule de la Charte incitait à " renouer la chaîne des temps ", cette rencontre a pu, en effet, en réactivant un épisode traumatique, être le point de départ d'une vocation d'historien chez un petit notable à l'appétit intellectuel que ni le maniement du papier timbré, ni l'administration d'une petite ville de province ne permettaient d'assouvir.

C'est peut-être la seule retombée bénéfique de la rencontre car, de sa proximité avec le marquis de Coislin et les milieux de l'ultra-royalisme,

45. Arch. dép. de Loire-Atlantique, 2 J 11. Lettre du 9 mars 1832.

46. Revenu d'émigration sous le Consulat, le marquis de Coislin avait rejoint l'armée des Princes en 1792. Pendant les Cent Jours, il reprit du service dans l'armée royale dite de la rive droite de la Loire. Chevalier de l'ordre de Saint-Louis en 1815, il fut élu la même année député de la Loire-inférieure. Réélu en 1816, il accéda à la pairie en 1823. Officier de la Légion d'honneur en 1825. Maréchal de camp depuis le 7/02/1816, il démissionna de ses fonctions en juillet 1830, mais finit par prêter serment pour conserver le bénéfice de la pairie. En 1832, lors de l'équipée de la duchesse de Berry, son château de Carheil ayant servi de lieu de réunion aux conjurés, il dut s'exiler à Jersey pendant trois ans.

47. Cf. supra, n. 15.

48. Arch. dép. de Loire-Atlantique, 2 J 5

49. Arch. dép. de Loire-Atlantique, 2 J 49 Attribué à un auteur anonyme, le brouillon de l'éloge funèbre est sans aucun doute possible de la main de Louis Jacques Marie Bizeul. 
L. J. M. Bizeul ne retira aucun avantage matériel. Pas plus les promesses du marquis que celles du baron François Auguste Fauvreau de Frenilly ${ }^{50}$ ne lui permirent d'obtenir la restitution, à son profit, de l'étude notariale nantaise de son beau-père. Au demeurant, l'homme n'était pas un carriériste. Lorsqu'il postule au poste de juge de paix de Blain au mois d'août 1842, tout en affirmant devant le procureur du roi n'être point "l'ennemi d'un gouvernement qui maintient l'ordre et le principe monarchique ", il ne peut cacher ses " sentiments de préférence et d'affection " pour le légitimisme, ce qui lui ôte toute chance d'obtenir la place ${ }^{51}$. Les termes qu'emploie alors L. J. M. Bizeul pour se situer politiquement sont précis et bien choisis : s'il est effectivement, intellectuellement, un homme d'ordre, son royalisme est, lui, affectif et sentimental. Nous avons vu qu'il était intimement lié à la maison de Coislin, il ne résistera pas à sa chute.

L'épilogue survint lors de la vente du château et la dispersion du mobilier. Dans ses Vieux souvenirs (1818-1848) ${ }^{52}$ publiés 52 ans après les faits, le prince de Joinville relata la visite éclair qu'il y effectua sous la conduite de L. J. M. Bizeul. De lui, il ne vit « qu'un vieillard poudré et à aile de pigeon, un type de vieux serviteur de l'ancien temps ${ }^{53}$ " à qui il prêta le comportement suivant :

"Puis il me regarda, je ne sais ce qu'il vit dans mes yeux, mais un accès de chagrin le saisit et c'est presque en larmes qu'il me prit pour confident de sa douleur de voir une des plus anciennes et des plus vénérables familles de Bretagne se fondre! [...]. Et ce vieil ami avait lieu d'être ému $[\ldots]^{54}$."

La description est cruelle. On peut toutefois s'interroger sur la véracité de ce récit sachant que l'achat du château de Carheil fut motivé par des raisons politiques. L'acquisition de ce haut lieu du légitimisme par le prince de Joinville, éminent représentant de la famille d'Orléans, signe la fin d'une époque. Elle a valeur de symbole et la fonction du passage incriminé est manifestement d'en restituer toute la portée. Sur la forme, les clichés convenus et la recherche du pittoresque abondent dans ce livre, sans trop d'égard, parfois, pour la réalité. Ainsi, visitant le château des Rohan à Blain, le prince écrit-il sans sourciller que sept des neuf tours du château furent détruites pendant la Révolution pour s'exclamer : " les Huns, les Sarrasins n'ont pas fait pis "! Il n'est donc pas exclu que la description qu'il donne de L. J. M. Bizeul n'ait été qu'un cliché de plus. Notons cependant que les sentiments qu'il lui prête, même dramatiquement exposés par un arti-

50. Le baron Fauvreau de Frenilly fut député du $4^{\mathrm{e}}$ arrondissement de la Loire-Inférieure (Savenay) de 1821 à 1827, date à laquelle il fut appelé à la chambre des pairs. Intime du comte d'Artois, il avait fondé avec François René de Chateaubriand, Louis de Bonald et Hugues Félicité Robert de Lamennais le journal Le conservateur (1818-1820).

51. Arch. dép. de Loire-Atlantique, 2 J 8. Brouillon de lettre, 21/08/1842.

52. JoINVILLE, prince de, Vieux souvenirs (1818-1848), Paris, Calmann Levy éditeur, 1894

53. Louis Jacques Marie Bizeul a alors 57 ans.

54. Joinville, prince de, Vieux souvenirs..., op. cit., p. 249 et Arch. dép. de LoireAtlantique, 2 J 5. Dans son journal, Louis Jacques Marie Bizeul confirme bien avoir fait visiter le parc du château, à cheval, au prince de Joinville, le 12 octobre 1842. 
fice littéraire, restent néanmoins plausibles, compte tenu de ce que nous savons de lui.

Dix années plus tard, au mois de juillet 1852, L. J. M. Bizeul est élu au conseil général de la Loire-inférieure. Pour ce scrutin, le préfet avait donné comme consigne à son administration de " n'appuyer ou tolérer comme candidats que des hommes sincèrement dévoués au Prince-président " mais que, si les exigences locales rendaient indispensables quelques candidatures légitimistes, il ne fallait accepter que « des hommes si près de se rallier à la cause napoléonienne que l'on puisse dès à présent les considérer comme ralliés ${ }^{55}$ ". Après l'élection, le préfet commanda un rapport confidentiel sur chaque nouveau membre. On peut y lire l'appréciation suivante :

"M. Bizeul est un archéologue distingué exclusivement voué à la science, il reste étranger aux débats politiques. Il jouit de l'estime et de la considération générale, mais la nature même de son caractère s'oppose à ce qu'il n'exerce jamais une influence ${ }^{56}$."

La fin de l'année 1842 aura donc marqué le point de départ d'un long cheminement au cours duquel L. J. M. Bizeul, qui s'était auparavant dépouillé de ses attributs de notable, commença à se défaire de sa fidélité au légitimisme pour ne plus se définir, exclusivement, que comme un savant archéologue. La création de l'Association bretonne, opportunément, offrit le cadre qui permit à cette transformation de s'opérer. La mise en évidence de cette trajectoire autorise un niveau de lecture plus intime, de son Aperçu général de l'étude des voies romaines : le passage singulier où il retrace son parcours de chercheur renvoie au moment où, remaniant son passé, il élabore pour lui-même un nouveau récit de vie qui fait sens. Désormais, il s'y identifiera complètement. C'est cette image que retiendra la postérité et qu'entretiendra l'histoire familiale. Voici comment, en 1887, Sévère Bizeul, un de ses petits-fils, transmit sa mémoire à Jacques Bizeul, son neveu ${ }^{57}$ :

" Notaire à Blain, sa passion pour l'étude et en particulier l'archéologie ne tardèrent pas à le dégoûter des devoirs de sa charge. C'était bien plus un bénédictin laïque heureux dans la solitude de sa bibliothèque, un charmant causeur à l'heure des récréations et dans les soirées de famille, un vaste et profond savant dans les congrès, qu'un de ces retors au feu sacré de la chicane, créé pour faire fortune en grattant du papier timbré ${ }^{58}$."

\section{J. M. Bizeul : sa formation.}

\section{Le foyer culturel nantais et ses figures tutélaires}

L'étude de sa correspondance érudite montre que les premiers documents attestant des recherches personnelles menées par L. J. M. Bizeul

55. Arch. dép. de Loire-Atlantique, 3 M 238. Lettre du 8 juillet 1852.

56. Arch. dép. de Loire-Atlantique, 3 M 236.

57. Musée de Blain. Carton Bizeul.

58. Musée de Blain. Carton Bizeul. Lettre de Sévère Bizeul à son neveu Jacques Bizeul, 1887. 
dans le domaine de l'histoire et de l'archéologie remontent à $1818^{59}$. À cette date, c'est un homme fait - il a 29 ans - et, semble-t-il, bien installé, qui entre en relation avec Pierre Louis Athénas, l'éminent savant de la Société académique du département de la Loire-Inférieure. Dans la lettre qu'il lui adresse, il sollicite la communication de son mémoire sur l'emplacement de Brivates portus et précise : "Sa lecture me fournira des points de reconnaissance qui seront en rapport avec des recherches dont je m'occupe à mon loisir. "

Nous retiendrons cette année comme point de départ, d'autant que, plus tard, L. J. M. Bizeul, faisant implicitement référence au début de ses recherches, crut pouvoir écrire :

" Blain n'a donc eu aucune histoire antérieurement au IX ${ }^{\mathrm{e}}$ siècle; et ce n'est que vers 1818 qu'on a songé à en faire la recherche, non pas dans les livres, mais dans le sol, suivant cet axiome si vrai, que le savant M. de Petigny ${ }^{60}$ n'avait pas encore écrit : "L'histoire de la Gaule n'est pas dans les bibliothèques; elle est dans le sol"61."

L'année 1818 a ceci de particulier qu'elle voit, au plan local, la renaissance de la Société académique de Nantes et, à l'échelle nationale, le lancement d'une grande enquête visant à développer les recherches archéologiques en France. Dans cette conjoncture éminemment favorable, L. J. M. Bizeul comprend que les travaux personnels qu'il mène pour tromper son ennui peuvent s'insérer dans une perspective plus vaste et lui donner accès à une aventure intellectuelle collective.

\section{Le foyer culturel nantais dans le contexte des années 1816-1824}

Sous l'Empire, la vie intellectuelle à Nantes était dominée par la Société des sciences et des arts du département de la Loire-Inférieure, continuatrice de l'Institut départemental des sciences et des arts fondé à Nantes le $1^{\text {er }}$ fructidor an $\mathrm{VI}^{62}$. Par sa formation, son positionnement social, L. J. M. Bizeul avait vocation à intégrer ce réseau. À vrai dire, depuis plusieurs années déjà, dès la fréquentation de l'École centrale, il avait été en contact avec quelques-uns de ceux qui, plus tard, fondèrent l'Institut; Pierre Marie Renou fut son professeur de rhétorique, P. L. Athénas présida le jury

59. Arch. dép. de Loire-Atlantique, 2 J 11. Brouillon de lettre de Louis Jacques Marie Bizeul à Pierre-Louis Athénas du 9 mai 1818.

60. Il s'agit de François Jules de Petigny de Saint-Roman (1801-1858), archéologue et historien, membre de l'Académie des inscriptions et belles-lettres.

61. BizEul, Louis Jacques Marie, "Des Namnètes aux époques celtique et romaine (époque romaine) ", Chapitre II. Bulletin de la Société académique de Nantes, tome 1, 1859, p. 153.

62. Sur la Société académique de Nantes, voir Blanloeil, Catherine, "De l'Institut départemental à la Société académique de Nantes et de la Loire-Inférieure : une société savante de province au XIX ${ }^{\mathrm{e}}$ siècle ", thèse université de Nantes, Jean Dhombres dir., 1992; RochCONGAR, Yves, Des hommes d'utilité publique. Une société savante au XIX ${ }^{\mathrm{e}}$ siècle, Nantes, Coiffard édition, 2010; GuIOMAR, Jean-Yves, Le Bretonisme. Les historiens bretons au XIX ${ }^{\text {e }}$ siècle, Mayenne, 1987. 
lors de son examen final. Quant à Richard jeune, un autre fondateur également examinateur, il donna en 1802 une communication sur les antiquités romaines de Nantes lors de la première séance publique annuelle de la Société des sciences et des arts, à laquelle L. J. M. Bizeul assista. Une fois installé à Blain, il cultiva de bonnes relations avec certains de ses membres présents dans la commune ${ }^{63}$.

Toutefois, entre 1816 et 1818, la perspective de son intégration à la société fut gravement compromise par l'action du vicomte de Cardaillac ${ }^{64}$, le commissaire spécial de Nantes chargé de l'épuration de l'administration locale après les Cent Jours. Persuadé qu'elle était un repaire de partisans de "l'usurpateur ", il s'était mis en tête de la dissoudre purement et simplement. Dans tout le pays en effet, depuis l'automne 1815, le rétablissement des cours prévôtales, une loi de sûreté générale et une autre contre les cris et écrits séditieux donnaient une base légale au déploiement d'une " terreur blanche " dirigée contre les anciens soutiens de Napoléon $1^{\mathrm{er}}$. Il fallut toute la détermination du président Fréteau et l'habileté du préfet de Brosses pour que, auto-épurée et reconstituée sous le nom de Société académique du département de Loire-inférieure, elle tienne sa première séance publique le 28 janvier 1818.

Le hasard voulut que la résurrection de la société savante coïncidât avec le lancement de la grande enquête initiée par l'Académie des inscriptions et belles-lettres dans le but de rassembler les matériaux pour servir à l'édition d'une nouvelle collection appelée Antiquités nationales. Elle fut officiellement lancée par une circulaire du comte Decazes, ministre de l'Intérieur, le 8 avril 1819. Sans remonter jusqu'au questionnaire de l'Académie celtique $^{65}$, on peut lui trouver un précédent dans la circulaire MontalivetLaborde de 1810. Adressée à tous les préfets, cette dernière visait à dresser un inventaire des abbayes, châteaux, tombeaux qui avaient survécu à l'épisode révolutionnaire. Mais, « au bout d'un an on cessa toute correspondance sur cet objet et on ne parut plus s'en occuper ${ }^{66}$ ".

La mécanique fut relancée lorsque, le 8 juin 1818, le ministre informa l'Académie des inscriptions et belles-lettres de son intention de lui remettre les mémoires issus de l'enquête de 1810. Après les avoir examinés, l'Académie voulut se saisir de cette opportunité pour parachever le travail commencé huit ans plus tôt. Elle rédigea donc un rapport à l'intention

63. Joseph Marie Rapatel et A. J. Cottin de Melleville, ingénieurs venus à Blain lors du creusement du canal de Nantes à Brest, François René Dubuisson, conservateur du cabinet d'histoire naturelle qu'il reçut chez lui en août 1816, le docteur Couétoux, conseiller municipal de Blain et correspondant local de la société.

64. Nommé en octobre 1815, Cardaillac resta en fonction jusqu'en décembre 1816.

65. Ozouf, Mona, "L'invention de l'ethnographie française : le questionnaire de l'Académie celtique ", Annales. Économies. Sociétés. Civilisations, 1981, n 2, p. 210-230.

66. "Rapport de la Commission chargée de l'examen des Mémoires envoyés à l'Académie par Son Ex. le Ministre de l'Intérieur " présenté le 20 novembre 1818. Histoire et mémoires de l'Institut royal de France : classe d'histoire et de littérature ancienne, t. 7, 1824 , p. 7-17. 
du ministre où figuraient plusieurs propositions " tendant à donner plus d'extension aux recherches archéologiques en France et à solliciter du Ministre des mesures relatives à la conservation des monuments dans chaque département ". Entérinées par le Ministre, elles débordaient en fait largement du cadre fixé en 1810 puisqu'elles concernaient désormais, et c'était une nouveauté, "les monuments Grecs, Romains, Gaulois, les tombeaux, les épitaphes, les titres, les chartes, les chroniques, et enfin tout ce qui peut fournir des éclaircissements sur les traits principaux de nos annales, l'illustration des familles, les institutions de la patrie".

Rassemblées sous la double injonction de "rechercher et décrire ", les douze instructions qui complétaient le rapport fournissaient un cadre programmatique à la recherche des antiquaires et consacraient son ancrage local à l'échelle du département. Elles préfiguraient, comme le note justement Odile Parsis-Barubé ${ }^{67}$, la politique patrimoniale de la France qui sera celle de la monarchie de Juillet ${ }^{68}$.

Seule tentative un peu importante faite sous la Restauration pour développer les recherches archéologiques nationales, cette enquête fut pourtant brutalement interrompue au mois d'avril 1824. Elle n'aura donc duré que cinq ans. Un laps de temps qui peut paraître court mais qui fut néanmoins très fécond : ces années servirent en effet de révélateur de l'état des recherches archéologiques qui, bien qu'éphémères, connurent dans le département une incontestable embellie sous l'impulsion du préfet de Brosses $^{69}$. Elles virent également l'entrée remarquée de L. J. M. Bizeul dans le milieu assez fermé des antiquaires : le 8 octobre 1821, sous le double parrainage prestigieux de P. L. Athénas et d'É. Richer, il fut admis membre correspondant de la Société académique de Nantes.

\section{Édouard Richer : le déclic?}

Entré à la Société des sciences et des arts en 1813, Édouard Richer (17921834) était une figure importante de la Société académique. Son premier écrit publié, daté lui aussi de 1818, était un Essai sur l'origine des constellations anciennes ${ }^{70}$. En 1820, sur le point de faire paraître le premier fascicule de ses Voyages pittoresques dans le département de la Loire-Inférieure, il se rendit à Blain pour rencontrer L. J. M. Bizeul. À la suite de cette entrevue, il intégra ses notes sur les voies romaines sortant de Blain dans son Voyage à la forêt du Gâvre, assorties d'une appréciation très élogieuse sur

67. PARSIS-BARUBE, Odile, La province antiquaire..., op. cit., p. 76-89. À voir également, GERSON, Stéphane, The pride of place. Local memories and political culture in nineteenthcentury France, London, Cornell university press, 2003.

68. En décembre 1820 fut créé un prix national doté de trois médailles d'or récompensant les trois meilleurs mémoires départementaux. Le 22 février 1821 fut publiée l'ordonnance qui instituait l'École des chartes.

69. Voir à ce sujet : DANIEL, Jacques, Louis-Jacques-Marie Bizeul : l'archéologue..., op. cit.

70. RicHER, Édouard, Essai sur l'origine des constellations anciennes, Nantes, imprimerie de Mellinet-Malassis, 1818, 36 p. 
leur auteur ${ }^{71}$. Le succès de l'ouvrage permit à L. J. M. Bizeul d'accéder à une certaine notoriété qui lui ouvrit les portes de la Société académique. Ses recherches, jusqu'alors empiriques, se cristallisèrent dès $1822^{72}$ en un projet dont le cœur était l'étude des voies romaines. Par l'intermédiaire d'É. Richer, il entra alors en contact avec la Société royale des antiquaires de France qui publia dans le tome 7 de ses Mémoires, en 1826, un petit résumé de ses travaux sur les voies romaines sortant de Blain.

Figure 5 - Édouard Richer (Bibliothèque municipale de Nantes, ms 2504)

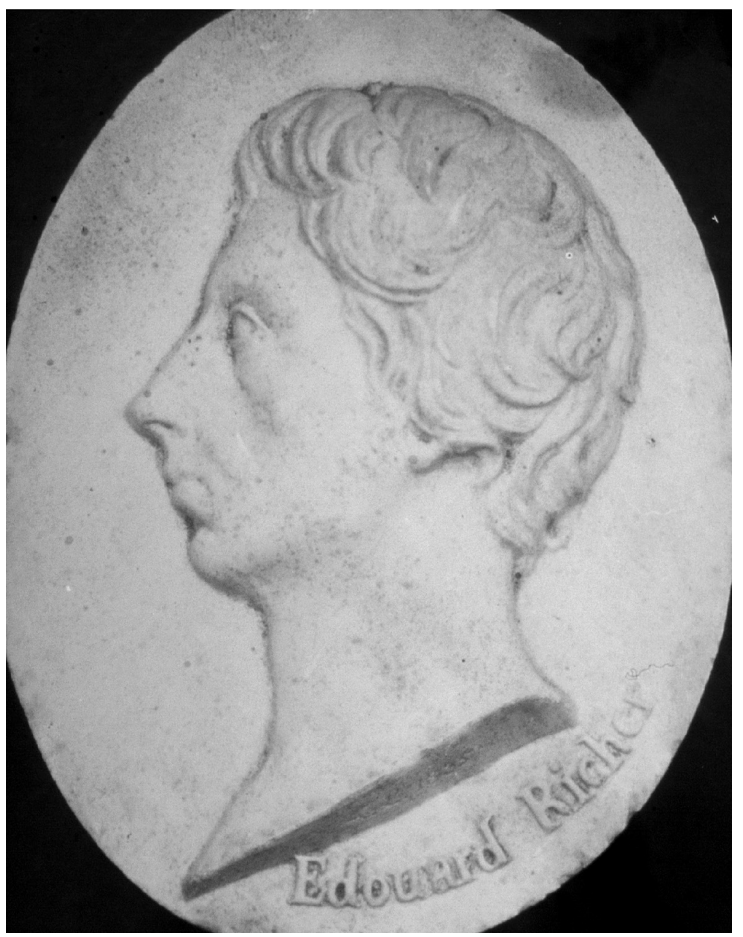

La rencontre avec É. Richer fit donc office de déclic. Mais elle fut éphémère, les approches des deux hommes étant, en réalité, fort différentes. É. Richer était venu à l'étude des antiquités par la poésie - celle d'Homère -, la littérature des débuts du romantisme - notamment les écrits de Bernardin de Saint-Pierre - et la philosophie spiritualiste. À l'instar de Charles Nodier et de Justin Taylor, auteurs des Voyages pittoresques et

71. RichER, Édouard, Voyages pittoresques dans le département de la Loire-Inférieure, Nantes, 1823, p. 56 et 81-83.

72. Le 24 février 1822 Louis Jacques Marie Bizeul fait paraître dans le Journal de Nantes et de la Loire-Inférieure, un article intitulé De l'ancienne Segora où il formule, pour la première fois, une méthode de localisation des villes antiques fondée sur l'étude du tracé des voies romaines. 
romantiques dans l'ancienne France ${ }^{73}$, il s'inscrit dans ce moment où, selon Odile Parsis-Barubé, le voyageur romantique, rencontrant l'antiquaire de province, dépasse " la posture méditative pour aboutir à la description précise d'un certain nombre d'édifices, ainsi qu'à l'identification des styles de construction ${ }^{74}$ ". Très bien informé, il parsème ses Voyages de nombreuses notations archéologiques mais il prétend décrire les paysages en combinant "l'imagination poétique qui se laisse facilement émouvoir ", "le jugement droit qui ne peut jamais être séduit ", le tout subordonné à cette " métaphysique transcendante qui nous aide à découvrir les rapports de l'homme avec l'univers ${ }^{75}$ ".

À l'opposé, L. J. M. Bizeul, sur les itinéraires qu'il suit le long des voies romaines, accumule les notes érudites : s'inscrivant dans la grande tradition des antiquaires, il traque les fausses interprétations et tente d'approcher une vérité qui ne laisse que peu de place à l'imagination. Esprit méthodique avant la lettre, il veut " apprendre le langage de la science avant de le parler ${ }^{76} "$.

L'entrée d'Édouard Richer dans le champ de l'étude des antiquités eut tout d'un météore. Très vite, dès 1826, son activité intellectuelle s'orienta en effet vers un mysticisme inspiré de la pensée d'Emmanuel Swedenborg ${ }^{77}$ qu'il contribua fortement à faire connaître en France. Aussi la rencontre entre les deux hommes se termina-t-elle par une rupture. L. J. M. Bizeul en fit l'aveu à Daniel Louis Miorcec de Kerdanet, l'érudit de Lesneven, avec qui il correspondait :

" Richer ne s'occupe plus d'études historiques; il s'est lancé à corps perdu dans le spiritualisme prétendu religieux; il publie en ce moment 6 volumes in $8^{\circ}$ dans lequel, dit-on, il développe ce nouveau système ${ }^{78}$; Dieu nous garde de le suivre dans la région élevée qu'il parraine : on y perdrait aisément la tête. Quant à lui, il en a été sérieusement malade et comme je l'ai grondé d'avoir joué sa santé contre des idées creuses et qu'il n'y a pas moins persisté, j'ai cessé à peu près de le voir ${ }^{79}$."

73. Les deux volumes consacrés à la Normandie sont strictement contemporains.

74. PARSIS-BARUBE, Odile, La province antiquaire..., op. cit., p. 128.

75. Richer, Édouard, Voyages pittoresques..., op. cit., p. 26.

76. Médiathèque de Nantes, ms 1983, lettre de Louis Jacques Marie Bizeul à Bottin, secrétaire de la Société royale des antiquaires de France, 30 août 1824.

77. Après avoir vécu des expériences mystiques, Emmanuel Swedenborg (1688-1772), scientifique, théologien et philosophe suédois, développa une théorie selon laquelle il existerait des correspondances entre monde matériel et monde spirituel, aux frontières incertaines. Édouard Richer fut un des premiers à introduire en France sa pensée, qui influença Charles Baudelaire (son poème Correspondances) et Honoré de Balzac (son roman Séraphîta). À Nantes, les adeptes de la pensée de Swedenborg se retrouvaient dans l'Église de la Nouvelle Jérusalem. Voir RICHER, Édouard, « De l'état actuel de l'esprit théosophique en Europe ", Le lycée armoricain, 1826, vol. 7, p. 135-152 et Notice sur Emmanuel Swedenborg et de l'état présent de la Nouvelle Jérusalem, Paris, Treuttel et Wurtz, 1835.

78. Richer, Édouard, De la Nouvelle Jérusalem, Paris, Treuttel et Wurtz, 1832-1835.

79. Arch. dép. de Loire-Atlantique, $2 \mathrm{~J}$ 11. Lettre à Miorcec de Kerdanet, 9 mars 1832. 


\section{Pierre Louis Athénas : le mentor}

"La découverte du chemin romain dont j'ai eu l'honneur de vous parler s'avance peu à peu. Je l'ai suivi cette semaine depuis Blain jusqu'auprès de Fégréac, d'où il se dirige sur Rieux. Il y a une branche qui prend au coin de Curun et se dirige sur Brivé. Quand j'aurai eu l'occasion de parcourir cette route dans toute sa longueur je vous ferai part, Monsieur, de mes remarques. Où pourrais-je me procurer la Table de Peutinger et l'Itinéraire d'Antonin? Ces ouvrages me manquent ${ }^{80}$."

C'est par ces mots que, dès 1818, L. J. M. Bizeul commença une correspondance suivie avec P. L. Athénas. Elle se poursuivit sans interruption pendant une dizaine d'années encore, jusqu'à la mort de ce dernier ${ }^{81}$. Sous ses yeux, les indices d'une présence gallo-romaine abondent. Avec l'aide de P. L. Athénas, il fera de l'étude des voies romaine, domaine jusque-là négligé, son thème de prédilection.

\section{Figure 6 - Pierre Louis Athénas. Château des ducs de Bretagne} (Musée d'histoire de Nantes, Alain Guillard)

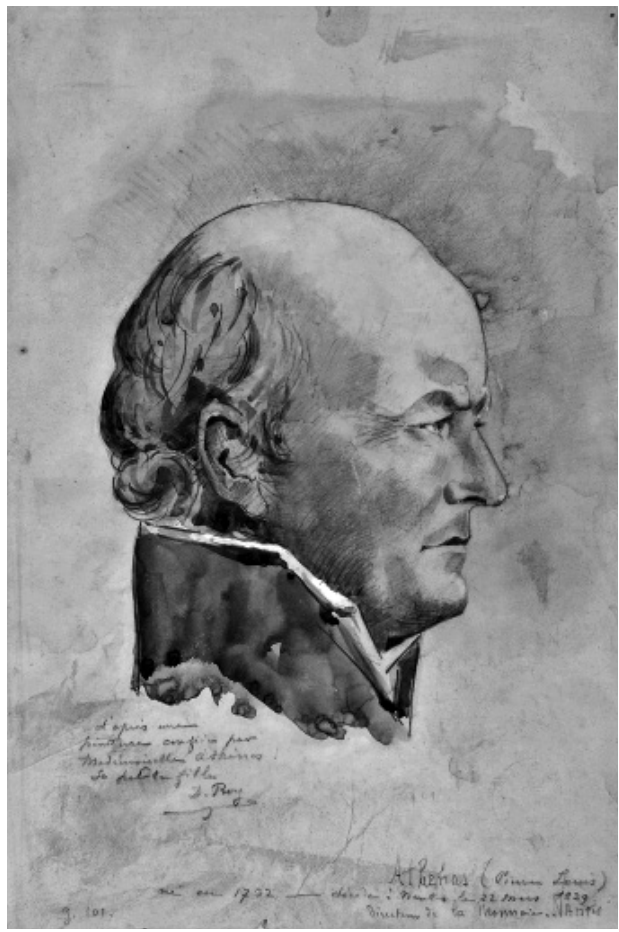

80. Arch. dép. de Loire-Atlantique, 2 J 11, lettre de Louis Jacques Marie Bizeul à Pierre Louis Athénas, 9 mai 1818.

81. Peu avant sa mort, Pierre Louis Athénas confia à Louis Jacques Marie. Bizeul ses manuscrits pour qu'ils soient édités. Ils furent cependant repris par ses héritiers qui s'occupèrent partiellement d'accomplir cette tâche. 
Pierre Louis Athénas (1752-1829) était, sans conteste, une figure dominante du foyer culturel nantais. Jeune chimiste formé à Paris, il était venu au Croisic en 1777 pour expérimenter un nouveau procédé d'extraction du sodium à partir du sel marin. Il s'intéressa ensuite à la production d'acide sulfurique et de colorants. Pendant la Révolution, qu'il accueillit favorablement, il fréquenta le club de la Halle. Élu à la municipalité de Nantes en 1791, du fait de ses talents de chimiste, il fut désigné par le Comité de salut public pour produire le salpêtre dont la " patrie en danger " avait besoin.

La période de la convention montagnarde lui fut moins favorable : jeté en prison pour avoir critiqué Robespierre, il faut sauvé par la réaction thermidorienne. S'ouvrit alors pour lui une carrière de notable ${ }^{82}$ que la Restauration ne parvint pas à enrayer : inquiété par le vicomte de Cardaillac pour son action pendant les Cent Jours, cet ancien partisan de l'Empereur put néanmoins reprendre place dans la Société académique qui, selon la formule de Camille Mellinet, « ne se composait que d'hommes d'ordre; mais ces hommes avaient la parole indépendante ${ }^{83}$ ".

Au sein de la Société académique, P. L. Athénas était sans doute l'un des derniers encyclopédistes encore actifs, capable à la fois d'intervenir sur des questions telles que les progrès de l'industrie et du commerce, l'amélioration de l'agriculture, ou encore la composition minéralogique des terrains, aspects de son activité généralement mis en avant par ses biographes. Son intérêt pour l'étude des antiquités et son action en faveur du patrimoine sont, en revanche, beaucoup moins documentés ${ }^{84}$.

Au mois de mai 1808, il avait présenté un rapport sur les découvertes réalisées lors des fouilles menées à Nantes par l'inspecteur voyer PierreNicolas Fournier (1747-1810) pendant les années 1805-1807, au nom d'une commission composée à cet effet ${ }^{85}$. Sans revenir sur les controverses interprétatives qu'elles suscitèrent ${ }^{86}$, signalons leur importance au regard de la naissance de l'archéologie à Nantes ${ }^{87}$. Elles conduisirent en effet leurs

82. Directeur de la Monnaie (1795-1817), secrétaire de la chambre de commerce (18031829), membre du conseil général (1800-1814), Pierre Louis Athénas fut en outre membre du jury de l'École centrale dès sa création (1795) et l'un des 31 fondateurs de l'Institut départemental de la Loire-Inférieure en 1798

83. Mellinet, Camille, Précis sur la Société académique depuis sa fondation en 1798 sous le titre d'Institut départemental jusqu'en 1833, Annales de la Société royale académique, 1833 , p. 457.

84. Rochcongar, Yves, Des hommes d'utilité publique..., op. cit, p. 48-49 et article "Athénas " dans Amouroux, Dominique, Croix, Alain, Guidet, Thierry, Guivarc'H, Didier (dir), Dictionnaire de Nantes, PUR, 2013, p. 65-66.

85. Procès-verbal de la séance publique de la Société des sciences et des arts du département de la Loire-Inférieure du 5 mai 1808, imprimerie Malassis, 1808, p. 55-87.

86. Celles, notamment, relatives à une dédicace à Voljanus, un dieu présenté comme topique, qui s'avéra en réalité être une dédicace à Vulcain. Voir MALigorne, Yvan, "Sanctuaires et structures vicinales dans deux chefs- lieux de cités de l'ouest de la Gaule (à propos de quatre inscriptions de Nantes et Angers) ", Armorica, Études sur l'ouest de la Gaule romaine, I, 2007, p. 55-71.

87. PIRAULT, Lionel, RouAud-RouAze Isabelle, "Les précurseurs de l'archéologie nantaise ", 303, n 50, 1996, p. 5-6. MontelL, Martial, SANTrot, Jacques, « La naissance de l'archéologie 
protagonistes à s'insérer dans la grande tradition portée par les antiquaires des siècles passés. Ainsi, P. N. Fournier consulta « les ouvrages de Grutter, de Leblanc, de Bouteroue, de Spon et d'un grand nombre des plus célèbres antiquaires, pour rapprocher leurs opinions sur des inscriptions et monuments analogues à ceux dont il faisait la découverte ${ }^{88}$ ". Notons également qu'à la même époque P. N. Fournier et P. L. Athénas devinrent membres correspondants de l'Académie celtique, faisant entrer la ville dans le premier réseau d'envergure consacré à l'étude des Antiquités nationales. De cette matrice, naîtra, en 1814, la Société royale des antiquaires de France et P. L. Athénas en fut également le correspondant. Au sein de la Société académique de Nantes, il apparaît donc comme porteur d'une histoire et d'une culture façonnées par la longue tradition antiquaire.

Lors de la reprise de l'enquête sur les antiquités nationales, il fut l'un des académiciens les plus actifs. Entre 1819 et 1824, les séances annuelles de la Société académique de Nantes et les livraisons du Lycée armoricain portent la marque d'une dizaine d'articles qu'il a rédigés sur des sujets allant de l'Antiquité romaine au Moyen Âge. Il s'intéressa, notamment, à la cathédrale, mais aussi aux variations du cours de la Loire et introduisit cette donnée nouvelle dans sa réflexion sur les localisations des anciens lieux ('île d'Her dans les marais de Donges en 843, état de la Loire au VII siècle à Basse-Indre et à Indret). Il disserta également sur la découverte d'un dépôt d'armes antiques (des haches, en réalité) trouvées à SaintJean-de-Boiseau, dans lesquelles il crut reconnaître le mattara gaulois que César mentionne dans le livre premier de ses commentaires ${ }^{89}$. Surtout, dès l'année 1818, il avait lu à la Société académique de Nantes un mémoire sur la situation du Brivates portus mentionné par Ptolémée dans sa Geographia. Récusant l'assimilation généralement admise avec Brest, il lui assigna, au terme d'un raisonnement croisant données étymologiques, critique textuelle et observations de terrain, l'endroit, dans la basse Loire, où la rivière Brivet se jette dans le fleuve. Ce mémoire, qui reste son œuvre majeure plusieurs fois rééditée ${ }^{90}$, connut une diffusion nationale et intéressa vivement, nous l'avons mentionné plus haut, L. J. M. Bizeul.

Son action en matière de défense du patrimoine mérite également d'être soulignée. Rédacteur du programme de l'Institut départemental des arts et des lettres en l'an VI, il avait indiqué, déjà, que s'il existait, "dans l'étendue du département, quelques monuments historiques, il serait important d'en faire la recherche, et la description pour tenter d'en connaître l'origine ".

à Nantes (1500-1860) ", Annales de Bretagne et des pays de l'Ouest, t. 118, n 3, sept. 2011, p. 41-44.

88. Procès-verbal de la séance publique de la Société des sciences et des arts du département de la Loire-Inférieure du 5 mai 1808, imprimerie Malassis, 1808, p. 86.

89. Séance publique annuelle de la Société académique de Nantes et du département de Loire-Inférieure tenue le 3 septembre 1821, Nantes, imprimerie Mellinet-Malassis, p. 79-82.

90. Athenas, Pierre Louis, "Mémoire sur la véritable position du Brivates portus de Ptolémée et sur le nom que portait Brest dans les premiers siècles de notre ère ", Mémoires de la Société royale des antiquaires de France, t. 3, 1821, p. 326- 356 et dans le Lycée armoricain, vol. 1, 1823, p. 145-155 et 217-229. 
Pionnier dans ce domaine, il ne pensait pas qu'il suffisait, comme le préconisait l'instruction jointe à la circulaire du comte Decazes, de « rechercher et décrire " les objets patrimoniaux. Encore fallait-il que l'État et les conseils généraux puissent les sauver de la destruction. Une exigence qu'il formula très tôt, dès le mois de juin 1819 :

«Des compagnies de démolisseurs s'emparent de nos vieux monuments départementaux pour en vendre les débris; et si l'on n'y met ordre promptement, nous aurons l'apparence d'un peuple qui a perdu ses monuments antiques qui sont les preuves de l'origine des nations et leur véritable titre de noblesse ${ }^{91}$."

En 1823, il lança un Cri d'alarme contre le vandalisme destructeur de tous nos vieux monuments où il préconisait des mesures conservatoires, très en avance sur son temps comme, par exemple, décréter qu'un " propriétaire qui se proposerait de démolir un ancien édifice ne put l'entreprendre sans en prévenir l'autorité qui en ferait l'acquisition, ou, tout au moins, en ferait lever des plans ou des dessins ${ }^{92}$ ". Des prises de position d'autant plus remarquables qu'elles interviennent bien avant le célèbre pamphlet de Victor Hugo, Guerre aux démolisseurs, dont la première version fut publiée en 1825 .

Sur ce plan, sa plus grande réussite fut le sauvetage de la tour d'Oudon sur laquelle il attira l'attention de la Société académique ${ }^{93}$. Vendue pendant la Révolution à un aubergiste qui entreprit de l'utiliser comme carrière de pierre, ce monument que P. L. Athénas pensait, à tort, être du $\mathrm{IX}^{\mathrm{e}}$ siècle, ne dut sa conservation, dit-il, "qu'à la ténacité de son ciment " qui résista même à l'emploi de la mine. À la suite de son intervention, le conseil général et le préfet votèrent les fonds nécessaires à son acquisition et la vente au profit du département fut conclue le 12 janvier 1820. Fort de ce succès, il convainquit par la suite le préfet de faire dresser les plans du château de Machecoul.

Au sein du milieu culturel nantais, Athénas était donc, sans aucun doute, le seul à pouvoir transmettre à L. J. M. Bizeul la culture archéologique qui lui faisait défaut. Il lui fit découvrir des sources telles que la Table de Peutinger, l'Itinéraire d'Antonin ou encore la Notice de l'empire. Il lui fit également connaître un certain nombre d'ouvrages qu'il ne se privait pas de critiquer comme le Voyage dans le Finistère de J. Cambry ou - l'Archéologie armoricaine d'A. Maudet de Penhouet. Leurs échanges, empreints d'un respect mutuel, montrent qu'ils étaient tous deux d'excellents connaisseurs des auteurs anciens et qu'ils se consultaient souvent sur des questions de traduction ou d'interprétation.

En matière d'archéologie, on considère aujourd'hui que le bilan scientifique des travaux de P. L. Athénas est négligeable, beaucoup de ses interpré-

91. Arch. dép. de Loire-Atlantique, 146 T 2. Lettre de Pierre Louis Athénas au maire de Nantes, 22 juin 1819.

92. Séance publique annuelle de la Société académique, 1823.

93. Séance publique annuelle de la Société académique du 29 juillet 1819. 
tations étant, en effet, erronées. Cependant, en dépit de ses limites, il exerça une indéniable influence sur les recherches menées par L. J. M. Bizeul. Après la lecture du mémoire sur la position du Brivates portus, celui-ci commença à concevoir l'idée que l'observation du tracé des voies antiques était la clé de la localisation des agglomérations disparues, une approche qui lui tint lieu de fil directeur dans ses travaux. En 1835, par exemple, il écrivait au sous-préfet de Savenay, à propos de la voie de Blain à Brivet :

"[...] si on la suivait avec attention, peut-être nous conduirait-elle à l'emplacement du Portus-Brivates de Ptolémée, placé avec si peu de critique à Brest, et dont la situation a été irrévocablement fixée vers l'embouchure de la rivière du Brivé, dans la Loire, par le respectable et savant Athénas ${ }^{94}$ ".

Et plusieurs années encore après le décès du vieux savant, L. J. M. Bizeul ne cessa de regretter sa disparition en déplorant le vide dans lequel elle l'avait plongé :

" [...] depuis la mort de M. Athénas, je ne trouve plus autour de moi personne avec qui je puisse m'entretenir de nos chères antiquités. Et [...] Il y a trois ans que nous avons perdu à Nantes le seul homme de ce pays-là qui sut écrire sur nos antiquités en les soumettant à une juste critique : c'était monsieur Athénas [...] C'était lui qui m'avait engagé à recueillir des observations mais jusqu'à présent le temps m'a manqué pour les mettre en ordre ${ }^{95}$ " (c'est nous qui soulignons).

Car, après la disparition de P. L. Athénas, L. J. M. Bizeul ambitionna d'être reconnu comme son successeur au sein du petit groupe des antiquaires nantais. C. Mellinet, écrivant au maire de Nantes, le présenta comme "l'un de nos érudits qui ont les droits les mieux établis à l'héritage de réputation de $\mathrm{M}$. Athénas ${ }^{96}$ ". Il est en effet frappant de constater que ses premiers mémoires publiés entre 1833 et 1837 dans les annales de la Société académique de Nantes se placent dans la continuité des travaux du maître disparu ${ }^{97}$.

\section{Quand L. J. M. Bizeul remanie son passé : la place des figures tutélaires dans " l'Aperçu général sur l'étude des voies romaines "}

Dans l'Aperçu général sur l'étude des voies romaines, tel qu'il fut lu en 1844, L. J. M. Bizeul plaça délibérément É. Richer à l'origine de sa carrière

94. Bibliothèque municipale de Nantes, ms 1719. Lettre de Louis Jacques Marie Bizeul au sous-préfet Darthey, 16 juin 1835.

95. Arch. dép. de Loire-Atlantique, 2 J 11. Lettre à Miorcec de Kerdanet, 9 mars 1832.

96. Arch. dép. de Loire-Atlantique, 2 J 11. Lettre de Camille Mellinet au maire de Nantes, 21 janvier 1836.

97. Son " Mémoire sur un dépôt d'armes antiques trouvées dans les fouilles du canal de Nantes à Brest " (1833) qui concerne, en réalité, un dépôt de haches et son "De Conradianus et de l'ouvrage qu'on lui attribue sous le titre Descriptio utriusque britanniae " (1836) continuent les recherches d'Athénas. En revanche, dans son étude sur les "Voies romaines de Nantes à Angers et de Nantes vers Saumur ", déposée en 1832 mais publiée seulement en 1837, il fait preuve d'une plus grande originalité. 
d'archéologue et passa sous silence le rôle de P. L. Athénas qui, de son aveu même, avait joué un rôle central dans sa formation d'apprenti archéologue. Une discordance qui nous amène à nous interroger sur le moment dans lequel s'inscrit ce discours.

\section{P. L. Athénas dans " l'Aperçu général sur l'étude des voies romaines »: une curieuse absence}

En ce milieu des années 1840, le temps des précurseurs de l'archéologie en Bretagne était épuisé, au point qu'on ne voyait plus désormais que leurs défauts. Une nouvelle génération d'antiquaires archéologues était à l'œuvre qui déboulonnait leurs statues. Ainsi, à Vannes, Fr. M. Cayot-Délandre ternissait la réputation du chanoine Mahé (1760-1831) : au reproche, déjà ancien, qui lui était fait de n'avoir mentionné qu'une partie des monuments du Morbihan dans son Essai sur les antiquités du Morbihan ${ }^{98}$, il ajouta l'accusation, qui frôlait l'imposture, de ne pas avoir vu, lui-même, beaucoup de ceux qu'il décrivait.

P. L. Athénas, disparu en 1829, dont la figure, en dehors du foyer culturel nantais, n'était plus aussi attractive, fut également rudement contesté. Le 5 juin 1840, dans la séance de la Société pour la conservation des monuments historiques qui se tint à Rennes en présence d'A. de Caumont, A. de Kerdrel ${ }^{99}$ remit en cause l'assimilation des haches de bronze analogues à celles trouvées à Saint-Jean-de-Boiseau avec les matar gaulois. Quelques personnes lui firent alors remarquer " que ces haches ont été désignées sous ce nom par quelques archéologues, notamment par M. Athénas, dans le Lycée armoricain ${ }^{100}$ ". Au nombre de ces " quelques archéologues " se trouvait L. J. M. Bizeul, qui dans son Mémoire sur un dépôt d'armes antiques trouvées dans les fouilles du canal de Nantes à Brest ${ }^{101}$, avait repris, en des termes qui, aujourd'hui, peuvent surprendre, l'interprétation que P. L. Athénas avait faite des armes trouvées à Saint-Jean-de-Boiseau :

" [Athénas] a, le premier en France, démontré, avec la richesse d'érudition et l'admirable rectitude de jugement qui le distinguaient, que cet instrument de cuivre, sous la forme d'un marteau taillant, est le matar, arme particulière aux peuples de l'ancienne Gaule, et que les dépôts, plus ou moins nombreux qu'on en a découverts, ont eu pour motif la cessation de la guerre entre deux peuplades qui se faisaient leur traité de paix en enfouissant des armes dans le sein de la terre, leur mère commune et l'une de leurs principales divinités. Le dépôt dont j'annonce la découverte, confirme parfaitement, à mon avis, l'opinion de M. Athénas. "

Absent à cette séance, il n'eut pas à se positionner publiquement mais il est clair qu'il était également visé.

98. MAHE, Joseph, Essai sur les antiquités du Morbihan, Vannes, 1825

99. Audren de Kerdrel, futur contradicteur de Louis Jacques Marie. Bizeul, joua un rôle de premier plan dans l'Association bretonne.

100. Compte-rendu de la séance dans le Bulletin monumental, volume 6, p. 241.

101. Bizeul, Louis Jacques Marie, Mémoire sur un dépôt..., op. cit., p. 357 
Dans ce nouveau contexte intellectuel, pour les congressistes de l'Association bretonne, P. L. Athénas n'était donc plus une référence fiable. Il eut été sans doute maladroit de la part de L. J. M. Bizeul de se réclamer de son parrainage. C'est sans doute la raison pour laquelle il ne le mentionna pas comme son mentor. Cependant, exception faite de l'épisode du congrès de Rennes, il est aisé de constater que, même s'il prit ses distances avec certaines de ses affirmations, L. J. M. Bizeul témoigna à plusieurs occasions de sa grande considération pour le vieil érudit. Mieux, il rendit hommage à son " respectable maître et ami " pour avoir écrit le mémoire sur la situation du Brivates portus, une " dissertation trop peu connue " qui, nous avons tenté de le montrer, contribua fortement à la formation de sa pensée.

\section{É. Richer dans “ l'Aperçu général sur l'étude des voies romaines »: une curieuse mise en valeur}

Dans l'Aperçu général sur l'étude des voies romaines, L. J. M. Bizeul évoqua sa rencontre avec É. Richer en ces termes :

"En 1820, un explorateur bien regrettable et fort zélé, Édouard Richer, vint me demander des notes pour mon voyage pittoresque dans la LoireInférieure. Livré à d'autres études, j'avais observé quelques antiquités mais sans but et sans méthode. Richer me fit sortir de cette apathie. Il excita mon zèle. Je me mis à l'œuvre ${ }^{102}$."

Si les faits sont indubitablement exacts, leur mise en perspective l'est, en revanche beaucoup moins, d'autant que, plus tard, L. J. M. Bizeul présenta leur rencontre sous un jour nouveau, atténuant singulièrement sa portée :

"Édouard Richer, écrivain trop loué de son temps à Nantes par un petit cercle d'amis, mais auquel il ne faut pas refuser certain mérite, vint à Blain me demander des notes sur les antiquités du pays. Je m'en occupais fort peu alors; non pas que je n'en eusse déjà le goût, mais parce que ma profession de notaire me laissait peu de loisir. J'en savais donc fort peu, et Richer moins encore; car, dans son Voyage pittoresque de la Loire-Inférieure, dont il amassait les matériaux, il n'a dépassé en rien la portée des notes qui lui ont été fournies ${ }^{103}$."

Au moment où se tint le congrès de Rennes, Richer jouissait encore d'une notoriété certaine parmi les érudits. Il la devait en partie à son Précis de l'histoire de Bretagne ${ }^{104}$ que compléta une Histoire de Bretagne ${ }^{105}$ en trois

102. Bizeul, Louis Jacques Marie, "Aperçu général sur l'étude des voies romaines " Bulletin de l'Association bretonne, $\mathrm{n}^{\circ} 1,2^{\mathrm{e}}$ partie, 1849, p. 3 .

103. Bizeul, Louis Jacques Marie, "Des Namnètes aux époques celtiques et romaines " (époque romaine) Chapitre II. Bulletin de la Société académique de Nantes, tome 1, 1859, p. 153-154.

104. RICHER, Édouard, Précis de l'histoire de Bretagne, Nantes, imprimerie MellinetMalassis, 1821-1822.

105. RICHER, Édouard, Histoire de Bretagne, Nantes, imprimerie C. Mellinet, 1822, 3 vol., $8^{\circ}$. Ils sont intégrés dans RichER, Édouard, Euvres littéraires, publication posthume annotée 
volumes. Ces productions, au demeurant, n'eurent rien d'original dans la mesure où elles prolongeaient l'historiographie bénédictine, - celle, en particulier de dom Morice - qui, bien que contestée par une nouvelle génération d'historiens, conservait encore de nombreux partisans ${ }^{106}$. S'il faut lui reconnaître une certaine originalité cependant, elle est à rechercher dans son écriture. É. Richer, en effet, fit œuvre de création littéraire en traitant de la Bretagne comme d'un " lieu et thème essentiellement poétique $^{107}$ ". Une représentation qu'il ne cessera d'illustrer dans les colonnes du Lycée armoricain, cette revue littéraire et historique de qualité qu'il avait fondée avec l'imprimeur Camille Mellinet et dont il fut, en quelque sorte, l'âme. Dans la préface qu'il rédigea pour les Euvres littéraires d'Richer, É. Souvestre reconnut sa dette envers lui. À propos de ses Lettres d'un Armorique publiées dans le Lycée armoricain, il nota : "Pas une page n'a été écrite sur la Bretagne depuis dix ans qui ne doive quelque chose à ces lettres. Pour notre part, nous y avons puisé pour tout ce que nous avons dit sur la mythologie armoricaine dans Les derniers Bretons ${ }^{108}$. " En ce sens, nous rejoignons l'opinion de J.-Y. Guiomar qui voit dans É. Richer une des sources majeures du courant qu'incarneront, plus tard, É. Souvestre et le jeune Th.-H. de la Villemarqué ${ }^{109}$.

La Bretagne ne fut pas traitée uniquement comme un thème littéraire dans le Lycée armoricain. En l'absence d'un bulletin régulier de la Société royale académique de Nantes, cette revue popularisa auprès d'un public averti quelques-unes des grandes questions débattues par les antiquaires. Une controverse sur l'origine des Bretons y occupa ainsi une place centrale et mobilisa la plupart des grands érudits que comptait alors la Bretagne. Menée au moyen d'affirmations invérifiables, la controverse alimentait la production de représentations plus proches de nouvelles mythologies que d'un véritable savoir. Notons que L. J. M. Bizeul, qui effectuait ses premiers pas dans l'étude des antiquités, ne prit aucune part à cette querelle et, qu'en privilégiant la période de l'occupation romaine, il s'inscrivit même à contre-courant de cette mode intellectuelle.

Le Lycée armoricain fut donc le lieu où s'exprimèrent et se popularisèrent auprès d'un public, certes averti, une quête des origines et l'affirmation d'une certaine identité culturelle bretonne, qu'à Rennes, en 1844, beaucoup de congressistes partageaient. C'est probablement la raison qui conduisit L. J. M. Bizeul, à ce moment précis, à valoriser sa rencontre avec

par Camille Mellinet d'après les indications de l'auteur, Nantes, imprimerie C. Mellinet, 1838, vol. -IV.

106. Ce point sera développé plus loin.

107. GuIOMAR, Jean-Yves, Le Bretonisme..., op. cit, p. 27.

108. Souvestre, Émile, "Notice sur Édouard Richer ", préface des Euvres littéraires d'Édouard Richer publiées d'après les indications de l'auteur par Camille Mellinet, Nantes, 1838. p. 16.

109. Guiomar, Jean-Yves, Le Bretonisme..., op. cit, p. 27. Théodore Hersart de La Villemarqué est le représentant du "bretonisme " littéraire et un des protagonistes des congrès de l'Association bretonne. 
É. Richer, au prix, même, d'une certaine distorsion de la réalité. Sans doute crut-il alors habile d'invoquer la caution de ce précurseur d'un courant celtophile aux recherches qu'il menait... sur la période de l'occupation romaine de l'Armorique.

\section{J. M. Bizeul et les enjeux du $2^{\mathrm{e}}$ congrès de l'association bretonne}

La place que L. J. M. Bizeul réserva à Richer et à P. L. Athénas dans son aperçu sur l'étude des voies romaines montre, à l'évidence, qu'elle obéissait à des considérations tactiques. En va-t-il de même du singulier autoportrait qu'il dresse pour les congressistes?

Figure 7 - L. J. M. Bizeul en 1860

(ville de Nantes, bibliothèque municipale, estampe 32, Frank Pellois)

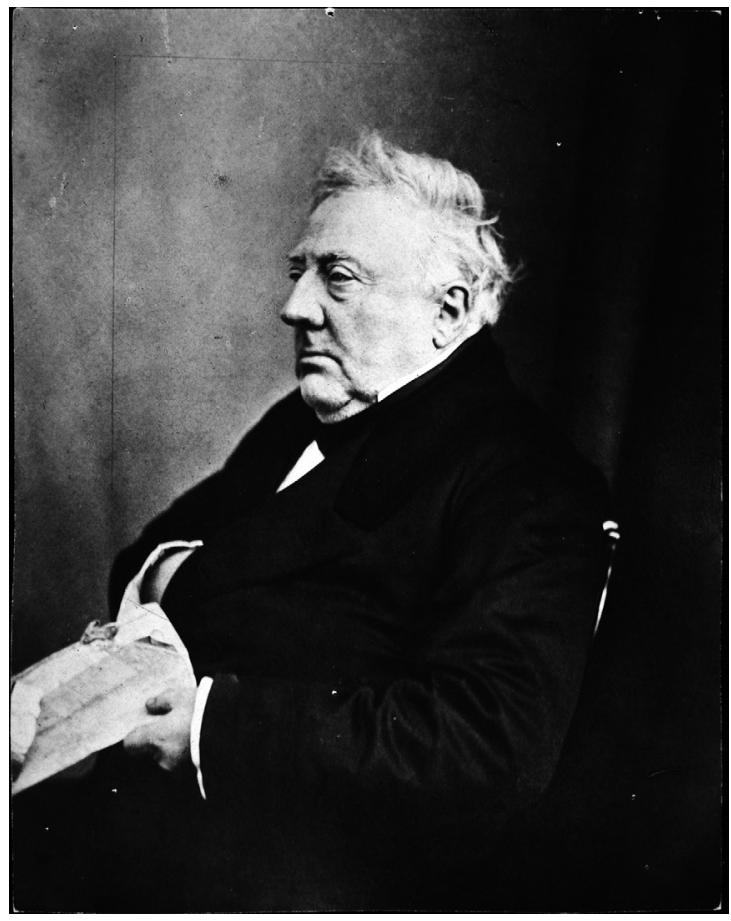

"Dans ce labyrinthe inextricable, j'ai cherché le fil conducteur, et j'ai cru le trouver dans l'investigation et l'étude sérieuse des voies romaines; j'ai cru que je retrouverais sur ces routes antiques, les traces du peuple conquérant et civilisateur, et qu'elles me conduiraient d'un établissement romain à un autre. J'ai fermé l'incomplète carte de Peutinger, l'itinéraire plus incomplet encore d'Antonin. J'ai osé ne pas relire la Notice des Gaules de D'Anville, ouvrage peu digne d'un si savant géographe, et je me suis lancé sur nos voies, cheminant lentement avec elles, la carte de Cassini et le crayon à la main. " 
Incontestablement, l'homme sait se mettre en scène : il forge l'image d'un savant en marche - littéralement - vers la connaissance et qui, simplement armé de son courage et de sa clairvoyance, trouve, en suivant à la trace les voies romaines, le sésame permettant d'ordonner en un ensemble intelligible le chaos né de la multiplicité des conjectures...

L'image est fascinante en ce sens qu'elle mêle l'humilité, associée à la seule quête de la vérité, à l'orgueil, légitime sans doute s'il est étayé, de vouloir être celui qui ouvre la voie. Elle aura de l'avenir. On la retrouvera, déclinée sur un mode épique, dans l'hommage posthume rédigé par $\mathrm{A}$. de la Noue ${ }^{110}$ comme dans la notice nécrologique, solidement étayée, que Prosper Levot lui consacra ${ }^{111}$. On la devine aussi à l'arrière-plan du portrait qu'à l'instigation de Benjamin Fillon, numismate vendéen réputé, le grand aquafortiste Charles Meryon exécuta en $1861^{112}$.

On aurait tort cependant de ne voir dans ce L. J. M. Bizeul par lui-même qu'une sorte d'autocélébration égocentrique. Plus fondamentalement, il faudrait s'interroger sur les raisons qui le poussent, à ce moment précis, à brosser ainsi son portrait.

Certes, dans une perspective que l'on peut qualifier de testamentaire, il invoqua son âge comme justificatif :

"Mon zèle existe encore, Messieurs; l'âge commence à emporter les forces. Mais je suis heureux de penser qu'une société que j'ai appelée de tous mes vœux pourra achever beaucoup mieux que moi ce que j'ai à peine ébauché., ”

Sans aller jusqu'à parler de coquetterie de sa part, il faut rappeler que, dans sa soixantième année, L. J. M. Bizeul est encore en bonne forme physique (les attaques de la goutte viendront plus tard) et que, surtout, il est intellectuellement très alerte. Plus sérieusement, il semble bien qu'à l'heure où s'ouvre une nouvelle page de l'histoire de l'archéologie en Bretagne, L. J. M. Bizeul, dans ce discours, prenne date pour l'avenir et cherche à se positionner par rapport aux enjeux du congrès.

110. La Noue, A. de, Hommage à la mémoire de M. Bizeul, doyen des archéologues bretons, Saint Brieuc. 1861.

111. LeVot, Prosper, " Notice nécrologique sur M. L. J. M. Bizeul de Blain ", Bulletin de la Société archéologique de Brest, t. 3, 1862-1863, p. 261-270.

112. Charles Meryon (1821-1868) est, en France, le plus célèbre aquafortiste de son temps. Il a su notamment, intéresser Charles Baudelaire et Victor Hugo avec ses eauxfortes de Paris où le réel, transfiguré, prend une dimension fantastique. À partir de 1858, il fait de fréquents séjours à l'asile psychiatrique de Charenton. Son portrait de Louis Jacques Marie Bizeul est devenu une icône jusque dans les années 1930. Il a été publié dans Fillon, Benjamin, Rochebrune, Oscar de, Poitou et Vendée : études historiques et artistiques, Fontenay-le-Comte, 1865. 
Figure 8 - Louis Jacques Marie Bizeul par Charles Meryon. Extrait de Fillon, Benjamin, de Rochebrune, Oscar

(Poitou et Vendée : études historiques et statistiques, Fontenay-le-Comte, 1865)

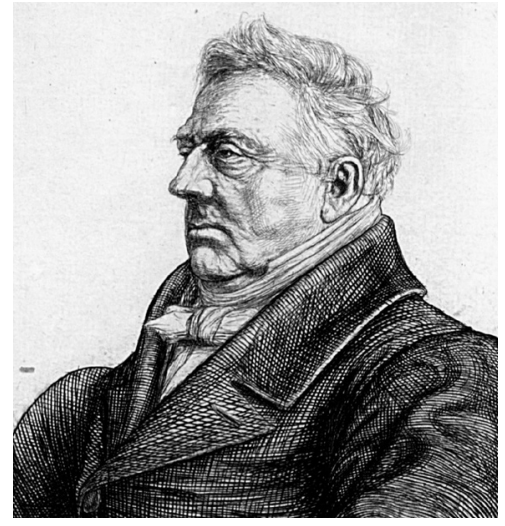

\section{Le positionnement de L. J. M. Bizeul dans le champ des études historiques bretonnes}

Jusqu'alors, ce résident et natif de Blain était surtout connu pour avoir publié, dans le bulletin de la Société royale académique de Nantes, dont il était membre correspondant depuis 1821, quelques études ponctuelles dont l'audience ne dépassait pas le cadre du foyer culturel nantais ${ }^{113}$. Toutefois, les échanges épistolaires qu'il entretenait depuis plusieurs années avec quelques-uns des grands érudits et antiquaires de l'Ouest armoricain lui avaient valu l'estime de ce milieu. Au point, par exemple, d'être sollicité par l'imprimeur A. Marteville et le professeur P. Varin, doyen de la faculté des lettres de Rennes, lorsqu'ils entreprirent de rééditer, en l'actualisant, le Dictionnaire historique et géographique de la province de Bretagne de Jean Ogée ${ }^{114}$. Si la contribution de L. J. M. Bizeul ne fut pas de premier plan, le fait mérite quand même d'être noté ${ }^{115}$. En effet, la ligne éditoriale de cette publication, en remettant en cause la réalité d'une colonisation de l'Armorique par les Bretons insulaires en l'an 383, à la suite du "tyran Maxime ", contestait par là même l'existence historique de Conan Mériadec qui lui était associée. C'était s'attaquer à une figure mythique, qui, quoique réfutée par le bénédictin de la congrégation de Saint-Maur

113. Il s'agit de son "Mémoire sur un dépôt d'armes antiques trouvées dans les fouilles du canal de Nantes à Brest " (1833) de son "De Conradianus et de l'ouvrage qu'on lui attribue sous le titre Descriptio utriusque britanniae " (1836) et de son étude sur "Les Voies romaines de Nantes à Angers et de Nantes vers Saumur ", déjà cités.

114. MARTEVILLE, A., VARIN, Pierre éd., Dictionnaire historique et géographique de la province de Bretagne dédié à la nation bretonne, (1777-1780), nouvelle édition, revue et augmentée, Rennes, 1843.

115. Commencée en 1839, la publication, sous forme de fascicules, s'acheva en 1843. La contribution de Louis Jacques Marie Bizeul s'arrête en mai 1842. 
dom Gui Alexis Lobineau ${ }^{116}$, tenait lieu de vulgate depuis sa reprise par dom Morice au milieu du XVIII ${ }^{\mathrm{e}}$ siècle $^{117}$. En ce sens, l'ouvrage fut un marqueur de l'émergence d'une nouvelle historiographie de la Bretagne et il n'est pas indifférent de constater que L. J. M. Bizeul en partageait les grandes orientations, comme il se plut à le souligner dans son discours devant le congrès de l'Association bretonne.

Ajoutons que le retentissement, dans le milieu des antiquaires, qu'eut son étude de 1841 sur les voies romaines de Bretagne ne tint pas seulement au fait qu'elle apportait des éléments inédits dans le domaine de la connaissance historique. Son mémoire, outre le fait qu'il comblait une lacune, portait en réalité un coup fatal au présupposé qui était à la base des conceptions d'un courant celtophile fort influent en Bretagne à la fin des années 1830, incarné principalement par Christophe Paulin de La Poix de Fréminville (1787-1848) ${ }^{118}$. Celui-ci, arguant de la faible présence, notamment en basse Bretagne, de vestiges romains recensés, prétendait en effet que l'occupation de l'Armorique par les Romains avait été superficielle et que celle-ci était restée une terre celtique. En commençant à lever le voile sur le dense réseau de voies parcourant l'Armorique (il dit en avoir repéré une quarantaine), L. J. M. Bizeul versait au débat, à l'encontre des celtophiles, des observations de terrain dont le poids devait se révéler décisif. Cela lui conféra, de fait, le rôle d'un chef de file qu'il résolut pleinement d'assumer.

\section{Une profession de foi archéologique}

Par l'accent mis dans son discours sur les observations de terrain, L. J. M. Bizeul se démarquait de la méthode utilisée par les cartographes du XVIII ${ }^{\mathrm{e}}$ siècle, lesquels, à l'instar du très respecté J.-B. Bourguignon d'Anville (1697-1782) ${ }^{119}$, ne raisonnaient essentiellement qu'à partir des indications chiffrées prises dans la Géographie de Ptolémée ou la Table de Peutinger pour restituer le tracé des routes et de la trame urbaine de l'Armorique romaine. Au-delà d'un simple plaidoyer pour le recours au terrain qui, de

116. LoBinEAu, dom Alexis, Histoire de Bretagne, 2 vol., Paris, 1707.

117. Dom Morice intègre dans son Histoire un mémoire de l'abbé Gallet, curé de Lamballe au XVIII ${ }^{\mathrm{e}}$ siècle qui plaçait Conan Mériadec aux origines de la Bretagne. MoRICE, dom Pierre Hyacinthe, Histoire ecclésiastique et civile de Bretagne, t. 1, Paris, 1750, précédé de 3 volumes de Preuves (1742, 1744, 1746). Interrompue par le décès de dom Morice, la poursuite de la parution fut assurée par TAILLANDIER, dom Charles, t. 2, Paris, 1756.Voir sur ce sujet : QUÉNIART, Jean, "Les mauriste et l'historiographie bretonne " dans TONNERRE, Noël Yves (dir), Chroniqueurs et historiens de la Bretagne, du Moyen Âge au milieu du XX ${ }^{e}$ siècle, Rennes, 2001, p. 111-123.

118. Fréminville, Chevalier DE, Antiquités de la Bretagne. Monuments du Morbihan, 1827, 1828, 1829. Deuxième édition revue et augmentée, Brest, Lefournier, 1834.Antiquités de la Bretagne. Finistère, 2 vol, Brest, Lefournier, 1832 et Brest, Come aîné, 1835.Antiquités de la Bretagne. Côtes-du-Nord, Brest, Lefournier, 1837.

119. AnVILle, Jean-Baptiste BouRGuIGNon D', Notice de l'ancienne Gaule tirée des monuments romains, Paris, 1760 . 
toute manière, était bien dans l'air du temps ${ }^{120}$, la critique de L. J. M. Bizeul prenait un tour radical dès lors qu'il récusait toute possibilité de construire une géographie ancienne selon une méthode mathématique, à partir de sources antiques dont il jugeait les interprétations pour le moins discutables. Arracher la localisation des villes antiques des mains des cartographes - lesquels comptent encore de nombreux partisans au sein des académies parisiennes ${ }^{121}$ - pour la confier à celles des archéologues, tel est bien, alors, son programme.

Notons cependant que ce retournement complet de la démarche, quoiqu'important sur le plan méthodologique, habillait aussi une posture : L. J. M. Bizeul s'affirmait comme un savant de province, homme de terrain, en rupture avec les élites parisiennes, celles, notamment, de l'Académie des inscriptions et belles-lettres, en qui il ne voulait voir que des hommes de cabinet. Ce faisant, L. J. M. Bizeul réactivait, en renouvelant son contenu, un vieil antagonisme Paris/province qui ne pouvait que recueillir un écho favorable parmi les membres de la nouvelle société savante de Bretagne. Le temps n'est plus très loin où il fera du baron Walckenaer, entomologiste de formation, secrétaire perpétuel de l'Académie des inscriptions et belleslettres depuis 1840, sa bête noire.

En résumé, attiré par l'étude de l'occupation romaine de l'Armorique - qu'il juge longue et profonde - plus que par celle de son passé gaulois ou celtique, critique par rapport à la réalité des émigrations bretonnes du IV $^{\mathrm{e}}$ siècle, L. J. M. Bizeul se présente en outre comme un archéologue de terrain, un authentique savant de province qui s'estime bien supérieur, par ses méthodes, aux chercheurs de salon des élites intellectuelles parisiennes. Clairement, il se place dans la grande tradition des antiquaires du XVIII ${ }^{\mathrm{e}}$ siècle dont il connaît les classiques. Son positionnement par rapport aux sources écrites fait écho à ce qu'écrivait B. de Montfaucon (1655-1741) sur le même sujet : "C'est une chose avérée que les marbres et les bronzes nous apprennent bien plus sur les funérailles que les anciens auteurs; et que les connaissances que nous puisons dans les anciens monuments sont bien plus sûres que ce que nous apprenons dans les livres ${ }^{122}$. " Quoique très fin connaisseur des textes historiques et des archives, il entend mon-

120. Sur les géographes de cabinet et les orientations de la Société de géographie fondée en 1821, voir les travaux d'Isabelle LABOULAIS, notamment, "Les géographes français de la fin du XVIII ${ }^{\mathrm{e}}$ siècle et le terrain, recherche sur une paradoxale absence " dans L'espace géographique, 2001/2 (vol. 30), p. 97-110.

121. Le baron Charles Athanase Walckenaer a fait paraître en 1839 une Géographie ancienne historique et comparée des Gaules, suivie d'une Analyse géographique des itinéraires des Gaules, où il écrit : "Pour pouvoir mettre à profit la géographie de Ptolémée, les itinéraires anciens et la table théodosienne, il est essentiel d'examiner comment ces ouvrages ont été composés, quelle est la cause des erreurs réelles ou apparentes qu'on y découvre et quels secours ils peuvent nous fournir pour déterminer mathématiquement la position des lieux antiques " (tome 3, XIII).

122. MontFaucon, Bernard de, L'antiquité expliquée et représentée en figures, Paris, 17191724, XV, 1, VI, tombeaux étrusques, p. 1 cité par CHOAY, F., L'allégorie du patrimoine, Paris, éditions du Seuil, 1992, p. 53. 
trer leurs limites et plaide, à travers l'étude des voies romaines, pour une reconnaissance de l'archéologie de terrain comme source irremplaçable de connaissance historique.

Tel quel, son discours est un plaidoyer pour la prise en compte de l'archéologie au sein de l'Association bretonne au moment où, tout juste admise, il lui faut asseoir sa place institutionnelle et préciser ses orientations. Secondairement, il remplit une autre fonction : se démarquer de la petite équipe - elle ne porte pas encore le nom de "bretoniste " - qui tente alors d'imprimer sa marque à la toute jeune association.

\section{Se différencier des «bretonistes »}

En l'absence d'Arcisse de Caumont, le deuxième congrès de l'Association bretonne à Rennes rouvrit un débat que le congrès de Vannes n'avait fait que clore provisoirement, en ayant admis l'archéologie en son sein mais en refusant de lui accorder une organisation spécifique. Cette fois-ci, l'offensive aboutit à la création d'une classe d'archéologie dotée d'une organisation, d'un bulletin et de moyens financiers propres. L. J. M. Bizeul qui s'y était opposé victorieusement lors du congrès de Vannes, y souscrivit cette fois, dans la mesure où le compromis maintenait, malgré tout, l'intégrité de l'association.

Ce changement avait été réclamé par, notamment, Aurélien de Courson, Audren de Kerdrel, Hersart de la Villemarqué, Aymar de Blois. Ils formaient alors le premier noyau de ceux qui plus tard, avec Arthur Lemoyne de La Borderie, se revendiquèrent " bretonistes ". Hormis leur intérêt pour l'histoire de la Bretagne/Armorique - et, accessoirement, leur adhésion au courant légitimiste - ils différaient, par leurs préoccupations, leurs pratiques, leur formation, de la voie que prétendait incarner L. J. M. Bizeul.

Au sein de ce groupe, les approches pouvaient être différentes - linguistique et littéraire pour Th. Hersart de la Villemarqué, historique pour A. de Courson ${ }^{123}$ - mais comme l'a bien montré J.-Y. Guiomar ${ }^{124}$, ils inscrivaient leur action dans un projet apologétique commun qui, par la régénération de la vision du passé d'une Bretagne pensée comme un conservatoire des traditions, visait à la glorification et à la restauration des bases de la civilisation chrétienne. Très inspirée par le courant traditionaliste et, notamment, par la pensée de J. de Maistre, L. de Bonald et F. de Lamennais, cette croisade était menée, entre autres, au moyen de la Revue de l'Armorique dirigée par A. de Courson, - sous-titrée " Dieu et la Société " - et dont le premier numéro, imprimé à Saint-Brieuc, sortit symboliquement le 15 août

123. Villemarqué, Théodore Hersart DE La, Barzaz-Breizh. Chants populaires de la Bretagne, 2 vol., 53 chants, Paris, Charpentier, 1839. Seconde édition publiée chez Delloye, 86 chants, 1845.Courson, Aurélien de, Histoire des origines et des institutions des peuples de la Gaule armoricaine et de la Bretagne insulaire depuis les temps les plus reculés jusqu'au $v^{e}$ siècle, Saint-Brieuc, 1843.

124. GuIOMAR, Jean-Yves, Le Bretonisme..., op. cit. 
1842, jour de l'assomption de la Vierge Marie vénérée comme la sainte patronne de la Bretagne.

Le bretonisme était partie prenante d'un mouvement européen, d'inspiration romantique à ses débuts, de révolte contre la place hégémonique réservée à la culture classique héritée de la Renaissance. Aussi, loin de partager le même intérêt que L. J. M. Bizeul pour l'occupation romaine, ses partisans avaient plutôt tendance à en minimiser les effets. Ce qui, parfois, les rendit proches des positions des celtophiles dont le principal représentant était alors le chevalier de Fréminville. Ils étaient enclins à n'y voir, tout au plus, qu'une parenthèse qui aurait masqué les continuités culturelles entre une Armorique, terre celtique primitive sinon primordiale, et la Bretagne médiévale chrétienne à ses débuts, objet de leur véritable préoccupation. Car leur projet était bien, en effet, de réconcilier celtisme et catholicisme au profit de ce dernier. Ils se distinguaient néanmoins des celtophiles par la place prépondérante qu'ils assignaient aux Bretons insulaires, lesquels, par leurs migrations, auraient implanté en Armorique une langue, un système d'organisation sociale hérité des Celtes et une forme de christianisme original, éléments fondateurs d'une identité bretonne.

En 1844, ils ne s'étaient pas encore démarqués du courant historiographique dominant qui se référait à dom Morice, et affirmaient que, dès le $\mathrm{IV}^{\mathrm{e}}$ siècle, s'était établi en Armorique, par voie de conquête, un royaume breton dirigé par Conan Mériadec ${ }^{125}$. A. de Courson venait d'ailleurs de sonner la charge contre l'article de P. Varin qui mettait en cause l'abbé Gallet et la mythique existence de Conan Meriadec ${ }^{126}$. Ce contexte polémique est essentiel pour comprendre les raisons qui ont pu pousser L. J. M. Bizeul, soucieux de se distinguer des bretonistes, à déclarer :

" [...] j'ai eu le malheur, je dois en faire ici ma confession, en lisant avec attention les dissertations de l'abbé Gallet, d'être amené à une conclusion entièrement contraire à celle de l'auteur ".

Au fond, les bretonistes, à la différence de L. J. M. Bizeul, cherchaient, autant qu'ils inventaient, les origines d'une nation, une préoccupation complètement étrangère à sa problématique de recherche. Cette divergence d'approche est naturellement centrale. Elle avait des implications méthodologiques. Dans son entreprise, L. J. M. Bizeul devait nécessairement s'appuyer sur la mise au jour de vestiges afin de prouver l'importance de l'occupation romaine. A contrario, les bretonistes spéculaient sur leur absence pour dresser le tableau d'une Armorique désertée par Rome dès la fin du IV ${ }^{\mathrm{e}}$ siècle. Les sources sur lesquelles ils s'appuyaient étaient essen-

125. C'est sous l'impulsion d'Arthur Lemoyne de La Borderie, à partir du congrès de l'Association bretonne de Saint-Brieuc de 1846, que s'effectuera le retour des bretonistes à dom Alexis Lobineau, avec comme corollaire l'abandon de la figure mythique de Conan Mériadec.

126. Courson, Aurélien DE, "Lettre de M. A. de Courson à M. Marteville, l'un des rédacteurs du Dictionnaire de Bretagne ", dans Marteville, A., Varin, P. (éd.), Dictionnaire historique..., op. cit., p. 261-263. 
tiellement textuelles, constituées principalement du corpus légué par les auteurs antiques, les chroniqueurs médiévaux et les historiens modernes mais aussi des documents d'archives qu'ils collectaient. C'était d'ailleurs la raison première de la présence en Bretagne d'A. de Courson qui, à l'instigation de Fr. Guizot, effectuait ce travail pour le compte du Comité des travaux historiques et scientifiques. Audren de Kerdrel, lui, était diplômé de l'École des Chartes. Quant à l'auteur du Barzaz Breiz, Th. Hersart de La Villemarqué, il battait la campagne et visitait les sacristies pour exhumer les chants populaires anciens censés receler, portés par la tradition orale, des informations historiques anciennes qu'il s'agissait de décrypter. En définitive, les bretonistes, dans leur système d'interprétation, accordaient plus d'importance à la langue - c'est par elle que les Gaulois, les Gallois ou les Bretons étaient assimilés aux Celtes - et aux documents d'archive - encore que, parmi les documents sur lesquels ils s'appuyaient pour le haut Moyen Âge, aucun n'était antérieur au IX ${ }^{\mathrm{e}}$ siècle - qu'aux vestiges.

L. J. M. Bizeul trace de lui, en creux, un autoportrait qui est leur exact opposé. S'abritant derrière la tradition antiquaire, il n'hésite d'ailleurs pas à forcer le trait en déclarant qu'il faut " laisser là les livres ", faisant mine d'opposer les textes à l'histoire "inscrite dans le sol ", alors qu'il a une très bonne connaissance de toutes les sources écrites. À ce stade, il ne fait qu'affirmer des convictions et exprimer des différences, encore feutrées, avec les bretonistes. L'affrontement ouvert, qui occupera une bonne partie des débats pendant toute la durée de l'Association bretonne, n'est certes pas encore déclaré mais on peut considérer que, dès le début de la vie de cette association, le décor en est déjà planté.

Dans la carrière savante de L. J. M. Bizeul, l'Aperçu général sur l'étude des voies romaines aura donc été un aboutissement, tout autant que le début d'une aventure qui le fera reconnaître par ses pairs comme le " doyen des archéologues bretons ".

Vers le mitan du XIX ${ }^{\mathrm{e}}$ siècle, le temps des précurseurs, héritiers des antiquaires solitaires et polyvalents du XVIII ${ }^{\mathrm{e}}$ siècle, s'efface. L. J. M. Bizeul, formé auprès de l'un d'eux en la personne de P. L. Athénas, perpétue ce que cette tradition a de meilleur. Mais, en même temps, il la dépasse par le caractère systématique de ses recherches. Au moment où, en Bretagne, avec quelque retard il est vrai, s'affirme le temps des sociétés savantes, il est à même de proposer, avec son travail sur les voies romaines de Bretagne, un véritable " projet collectif de recherche " avant la lettre.

Au sein de la classe d'archéologie de l'Association bretonne et de la Société archéologique et historique de Nantes ${ }^{127}$, L. J. M. Bizeul, qui, dans son parcours de vie, ne s'identifie plus désormais que comme un archéologue, promeut, face aux bretonistes qu'anime un projet apologétique,

127. Fondée en 1845 au congrès de Nantes de l'Association bretonne, elle est la filiale, en Loire-Inférieure, de sa classe d'archéologie. 
une archéologie de terrain qui, en dépit de ses limites propres à l'époque, entend faire la démonstration de sa validité par la preuve. Certes, dans l'Aperçu général sur l'étude des voies romaines, le " champ de l'Archéologie " n'est présenté que "sous le rapport de la géographie ancienne ". Pour autant, on peut considérer que son propos, d'une portée plus générale, annonce déjà les générations qui, à partir de la fin des années 1840, impulseront l'essor des fouilles archéologiques en Bretagne ${ }^{128}$.

Ce court texte apparaît bien comme un jalon à la charnière de la fin d'une époque et de l'avènement de nouveaux modes de pensée et d'organisation de l'archéologie en Bretagne. De ce point de vue il incarne le moment où, définitivement, l'archéologue prend le pas sur l'antiquaire

Figure 9 - Carte des voies romaines publiée dans le Bulletin monumental (tome IX, 1843) d'après les travaux de L. J. M. Bizeul

(Communiquée à A. de Caumont au congrès scientifique d'Angers du mois de juin 1841, elle n'intègre pas les compléments publiés dans l'Annuaire du Morbihan en 1842)

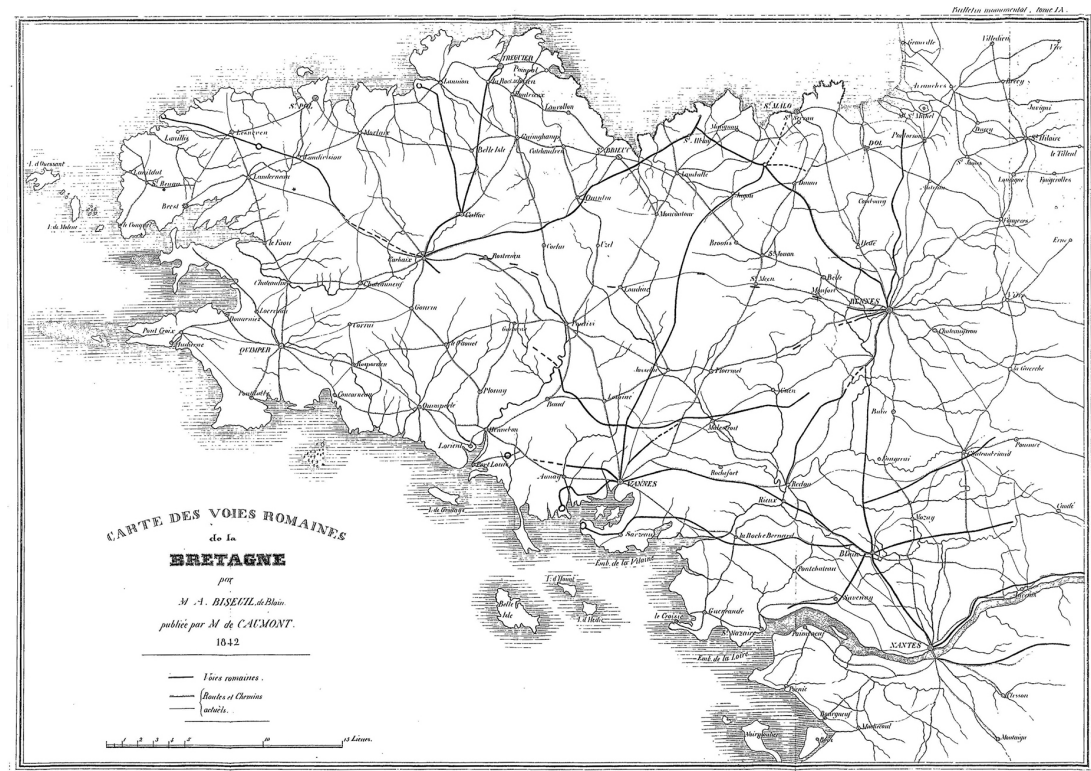

128. Voir, pour le Morbihan, Le Pennec, Christophe, " La Société Polymathique et la naissance de collections archéologiques en Morbihan ", Annales de Bretagne et des Pays de l'Ouest, 118-3, 2011, p. 73-96. 


\section{RÉSUME}

Louis-Jacques-Marie Bizeul (1785-1861) fut, sous la Restauration, maire de Blain, petite ville distante d'une trentaine de kilomètres de Nantes. Notaire de profession, il commença, dès la fin des années 1810, à s'intéresser à l'étude des antiquités. Au contact du foyer culturel nantais et, notamment, de PierreLouis Athénas (1752-1829) alors figure dominante de la Société académique de Nantes, il fit de l'étude des voies romaines son domaine de prédilection. Toutefois, ce n'est que tardivement, au début des années 1840, qu'il obtint la reconnaissance de ses pairs et s'affirma comme un érudit et un archéologue de premier plan au sein, notamment, de l'Association bretonne qu'il avait contribué à fonder aux côtés d'Arcisse de Caumont. Son Aperçu général sur l'étude des voies romaines, lu au congrès de Rennes (1844) de cette association, illustre ce tournant. Replacée dans son contexte, cette courte allocution, véritable profession de foi archéologique, se prête à différents niveaux de lecture : aboutissement d'une trajectoire personnelle, elle témoigne aussi du passage de témoin entre deux générations celle des " antiquaires " et celle des " archéologues " et préfigure déjà les affrontements entre " romanistes " et " bretonistes " qui émailleront l'existence de la première Association bretonne. À ce titre, elle peut être considérée comme un jalon important de l'histoire de l'archéologie en Bretagne.

\section{ABSTRACT}

During the Restoration, Louis-Jacques-Marie Bizeul (1785-1861) was the mayor of Blain, a small town about thirty kilometres north of Nantes. A notary by profession, he became interested, as early as the late 1810s, in the study of antiquities. In contact with Nantes's cultural society, and in particular Pierre-Louis Athénas (17521829) who was a prominent figure in the Nantes Academic Society, L.-J.-M. Bizeul made the study of the Roman roads his chosen field. However, it was not until the early 1840s that he won recognition among his peers and established himself as a scholar and a leading archeologist, in particular within the Breton Association that he had helped found along with Arcisse de Caumont. His Aperçu général sur l'étude des voies romaines, read during that association's conference in Rennes (1844), exemplifies this turning point. Back in context, this short address, a true archaeological profession of faith, lends itself to multiple readings: as the culmination of a personal journey, it also signalled the handover between two generations, that of the "Antiquarians" and that of the "Archaeologists", and already foreshadowed the clashes between "Romanists" and "Bretonists" which occurred throughout the history of the first Breton Association. As such, it can be considered an important milestone in the history of archaeology in Brittany. 